\title{
CFD Analysis of Energy Separation in Ranque-Hilsch Vortex Tube at Cryogenic Temperature
}

\author{
T. Dutta, ${ }^{1}$ K. P. Sinhamahapatra, ${ }^{2}$ and S. S. Bandyopadhyay ${ }^{1}$ \\ ${ }^{1}$ Cryogenic Engineering Centre, Indian Institute of Technology, Kharagpur 721302, India \\ ${ }^{2}$ Department of Aerospace Engineering, Indian Institute of Technology, Kharagpur 721302, India \\ Correspondence should be addressed to T. Dutta; tan_dat2003@yahoo.com
}

Received 30 April 2013; Accepted 11 September 2013

Academic Editor: Robert Spall

Copyright (c) 2013 T. Dutta et al. This is an open access article distributed under the Creative Commons Attribution License, which permits unrestricted use, distribution, and reproduction in any medium, provided the original work is properly cited.

\begin{abstract}
Study of the energy separation phenomenon in vortex tube (VT) at cryogenic temperature (temperature range below $123 \mathrm{~K}$ ) has become important because of the potential application of VT as in-flight air separator in air breathing propulsion. In the present study, a CFD model is used to simulate the energy separation phenomenon in VT with gaseous air at cryogenic temperature as working fluid. Energy separation at cryogenic temperature is found to be considerably less than that obtained at normal atmospheric temperature due to lower values of inlet enthalpy and velocity. Transfer of tangential shear work from inner to outer fluid layers is found to be the cause of energy separation. A parametric sensitivity analysis is carried out in order to optimize the energy separation at cryogenic temperature. Also, rates of energy transfer in the form of sensible heat and shear work in radial and axial directions are calculated to investigate the possible explanation of the variation of the hot and cold outlet temperatures with respect to various geometric and physical input parameters.
\end{abstract}

\section{Introduction}

The Ranque-Hilsch vortex tube (VT) separates a compressed gas stream into two lower pressure streams with one stream having higher temperature and the other having lower temperature than the inlet stream. This phenomenon is referred to as energy (temperature) separation. VT consists of one or more tangential inlet nozzles (vortex generator/generator), a tube, an orifice at the cold gas outlet, and a conical control valve at the hot gas outlet. When compressed gas is tangentially injected into the VT through the inlet nozzles, intense swirling flow is generated and the gas proceeds towards the hot outlet. A portion of the gas moving towards the hot outlet reverses its direction near the hot outlet and moves towards the cold outlet along the axial region (also called the core region) of the tube. The peripheral region of the flow is found to be warmer than the inlet gas, while the flow near the core region becomes colder than the inlet gas. The warm peripheral flow comes out through the annular space between the tube wall and the conical valve at the hot outlet. The colder core flow in the opposite direction comes out through the central orifice of the cold outlet. The conical valve placed at the hot outlet controls the relative mass flow rate of hot and cold gases. The airflow in a VT is shown in Figure 1(a) with representative values of pressures and temperatures at inlet and outlets for VT working with room temperature air. Due to its advantages, such as no moving parts, small size, lightweight, low cost, no maintenance, adjustable temperatures, and instant cold and hot air, VTs are widely used for low-temperature applications, such as cooling machining operations, cooling electrical and electronic control cabinets, cooling CCTV cameras, cooling soldered parts, cooling and dehumidifying gas samples, setting hot melts, testing thermostats, setting hot glue, cooling food, chilling environmental chambers, cooling heat seal, and so forth.

Ranque [1] invented this device and first reported the energy separation phenomenon. Later Hilsch [2] conducted comprehensive experimental and theoretical studies to improve the efficiency of VT. Since then, many experimental, analytical, and numerical studies have been carried out to investigate the energy separation mechanism and improve the performance of VT. Xue et al. [3] presented a summary of different explanations of energy separation found in the literature. Fulton [4] suggested that expansion of the inner 


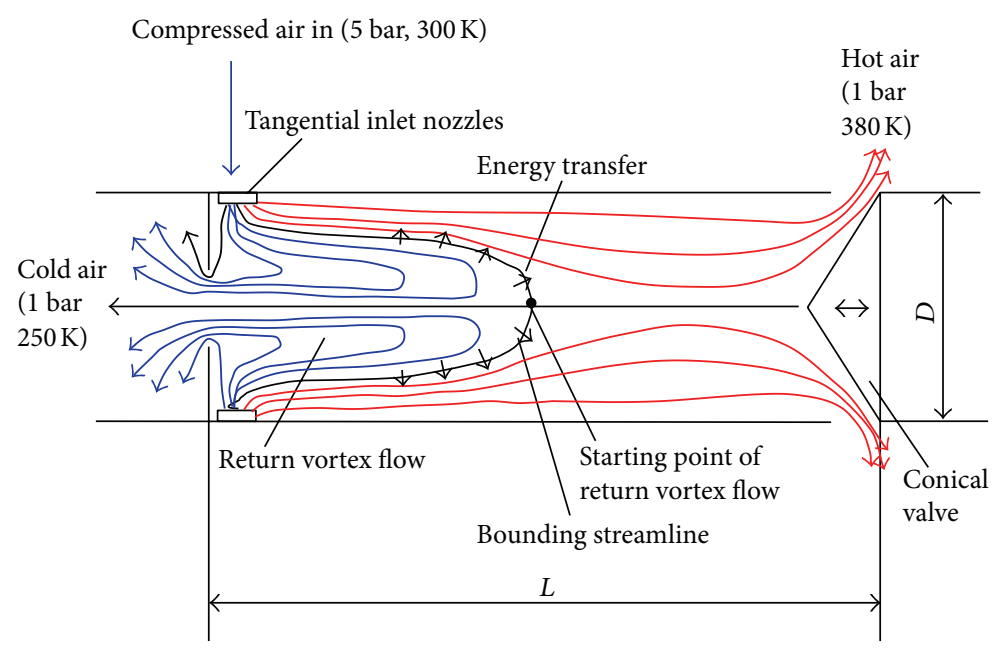

(a)

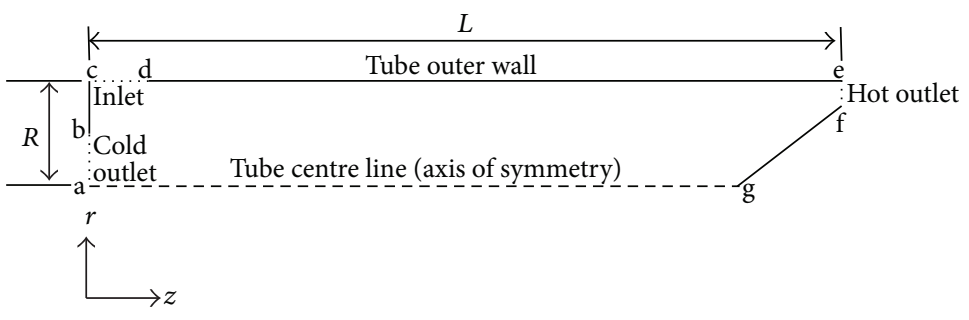

(b)

Figure 1: (a) Schematic flow pattern in vortex tube. (b) Working geometry of the vortex tube.

fluid layers and compression of the peripheral fluid layers was the cause of energy separation. Linderstorm-Lang [5] analytically determined the velocity and temperature fields inside the VT. Kurosaka [6] presented the theory of acoustic streaming to explain energy separation. Ahlborn and Groves [7] postulated the theory of secondary circulation in VT. Saidi and Valipour [8] experimentally studied the effects of different geometrical and thermophysical parameters and moisture content in the air on energy separation. They observed that the energy separation and efficiency decrease with increase in moisture content in the inlet air. Aljuwayhel et al. [9] investigated the energy separation mechanism using the FLUENT code with standard $\kappa-\varepsilon$ and RNG $\kappa-\varepsilon$ turbulence model. Behera et al. [10] presented a CFD model to investigate the effects of different geometrical parameters on temperature separation using the STAR CD code. Kazantseva et al. [11] used the software CFX-TASKflow to numerically solve the Navier-Stokes equations and observed the flow patterns and the velocity and temperature fields in a VT. Eiamsa-ard and Promvonge [12] used a numerical model for the simulation of energy separation effect. They used the Algebraic Reynolds Stress Model (ASM) and $\kappa-\varepsilon$ turbulence model and concluded that the diffusive transport of mean kinetic energy had a substantial influence on energy separation. T. Farouk and B. Farouk [13] used the large eddy simulation (LES) technique to predict the energy separation. Eiamsa-ard and Promvonge [14] analyzed the energy separation in a uniflow VT by considering the effects of turbulence models (ASM and $\kappa-\varepsilon$ model) and numerical schemes (first and second order upwind, hybrid). They obtained reasonably good agreement of the static and total temperatures with the experimental data. The ASM was observed to provide marginally better prediction of the profiles than the $\kappa-\varepsilon$ model. Farouk et al. [15] used an axisymmetric CFD model with the LES technique to predict the temperature separation and the separation of nitrogen-helium mixture in a VT. The species separation was attributed to the Soret diffusion. Secchiaroli et al. [16] presented a CFD model using FLUENT. They used an axisymmetric grid for the RNG $\kappa-\varepsilon$ turbulence model and a 3-D grid for conducting simulations with LES. Dutta et al. [17] compared the influence of different Reynolds Averaged Navier-Stokes (RANS) based turbulence models in predicting the temperature separation using FLUENT. Dutta [18] carried out a similar study with $2-\mathrm{D}$ as well as $3-\mathrm{D}$ numerical simulations of energy separation using different RANS turbulence models. Results obtained by Dutta [18] showed that temperature separations predicted by 2-D and 3-D models are very close to each other. Shamsoddini and Nezhad [19] performed 3-D computation using FLUENT to study the effects of the number of nozzles on the flow and cooling power of VT. They observed that the cooling power increased by $8.7 \%$ and $T_{c}$ decreased from $-0.2^{\circ} \mathrm{C}$ to $-1.28^{\circ} \mathrm{C}$ when number of nozzles increased from 2 to 8 . Eiamsa-ard et al. [20] used a cooling water jacket to directly cool the hot tube of a VT and observed higher cold temperature separation and cooling efficiency compared to the VT without cooling. 
They suggested that the use of cooling water jacket improves heat transfer from the axial region to the peripheral region, which leads to higher cooling efficiency. Simões-Moreira [21] developed a "thermodynamic air standard cycle" for VT and found it to be a highly irreversible device. According to him, all VTs operate between the isentropic/ideal condition and the adiabatic throttling process for a given inlet state. Kocabas et al. [22] developed the ANN and multilinear regression models of energy separation. Dutta et al. [23] carried out 3-D CFD analysis of energy and species separation phenomena in VT using the NIST real gas model.

The majority of available literature is focused on energy separation with gaseous air at normal atmospheric temperature as the working fluid. However, recent studies [2428] reflect that the VT working at cryogenic temperature (temperature range below $123 \mathrm{~K}$ ) with liquid-vapour twophase air has the potential to separate air into its main constituents: oxygen and nitrogen. When compressed twophase air at cryogenic temperature is injected into the VT, energy separation as well as mass transfer takes place inside the tube. As a result, oxygen rich fluid stream, which is predominantly in liquid phase, comes out from the hot outlet, and nitrogen rich fluid stream, which is mainly in vapour phase, comes out from the cold outlet. Because of this possibility, VT has become a potential candidate for use in an in-flight air collection and enrichment system (ACES) of air breathing propulsion $[26,27]$. The ability of VT to produce high purity oxygen (greater than 90\%) from air at cryogenic temperature and other features of VT such as low weight, small volume, absence of any moving part, and minimum maintenance requirement makes it an attractive option for space application [24]. The huge potential for application of VT in ACES indicates the necessity of detail theoretical analysis of VT at cryogenic temperature. However, before analyzing the flow phenomenon in a cryogenic two-phase $\mathrm{VT}$, it is important to analyze the energy separation in a VT operating with gaseous air at cryogenic temperature. But, there is hardly any published literature on numerical analysis of either single-phase or two-phase flow in VT at cryogenic temperature.

In the present work, a CFD model is used to investigate the energy separation in the VT with gaseous air at cryogenic temperature as the working fluid using the CFD software FLUENT 6.3.26. Since the published literature on experimental or theoretical investigation on energy separation at cryogenic temperature is scarce [28], the CFD model presented in our previous work [17] for the VT operating with gaseous air at normal atmospheric temperature is used here. The flow parameters inside the tube are analyzed to investigate the energy separation mechanism. Also, a CFD based parametric analysis is carried out at cryogenic temperature by observing the variation of the hot outlet and cold outlet temperatures with respect to various geometric and physical input parameters in order to optimize energy separation. It may be noted that in the present study, the term "temperature" indicates "total temperature" unless otherwise specified.

\section{Numerical Model}

In our previous work [17], an axisymmetric CFD model was used to carry out CFD simulation of energy separation in VT with air at normal atmospheric temperature. The study shows that the CFD model predicts $T_{h}$ and $T_{c}$ with reasonable accuracy. Four different RANS based turbulence models, (standard $\kappa-\varepsilon$, RNG $\kappa-\varepsilon$, standard $\kappa-\omega$, and SST $\kappa-\omega$ ) were used in the CFD model, and $T_{h}$ and $T_{c}$ predicted by using the standard $\kappa-\varepsilon$ model were found to be the closest to the experimental data compared to $T_{h}$ and $T_{c}$ computed by using other three turbulence models. Similar observation was made by Dutta [18] also in the 2-D and 3-D numerical simulations of energy separation using different RANS turbulence models. Since the working fluid in the present study is gaseous air at cryogenic temperature, the CFD model presented earlier by Dutta et al. [17] is used in the present study with only the standard $\kappa-\varepsilon$ turbulence model and the values of the fluid properties appropriate for cryogenic temperature are considered. Since experimental or theoretical work on energy separation at cryogenic temperature is scarcely reported in the open literature, the results of present CFD analysis at cryogenic temperature could not be validated.

The VT is approximated into an axisymmetric computational domain as shown in Figure 1(b). The length $(L)$ of the $\mathrm{VT}, \mathrm{c}-\mathrm{e}$, is $120 \mathrm{~mm}$ and the radius of the $\mathrm{VT}$, a-c, is $6 \mathrm{~mm}$. The dotted line, a-g, is the axis of symmetry. The inlet boundary is represented by $c-d$ and the line, a-b, is the cold outlet boundary having a radius of $3.5 \mathrm{~mm}$. The dotted line, e-f, is the hot outlet boundary. The hot outlet opening is varied to control the hot outlet mass fraction, $\xi$ (ratio of mass flow rates at hot outlet and inlet).

Mesh in the computational domain is generated by Gambit 2.3.16 with refinement of meshes near the wall. The boundary conditions are as follows:

(i) stagnation boundary condition at the inlet is specified with total pressure of $0.5422 \mathrm{MPa}$ (abs) and total temperature of $115 \mathrm{~K}$. Angle between the radial velocity component and resultant velocity is considered as $75^{\circ}$;

(ii) pressure boundary condition at hot outlet with static pressure of $0.15 \mathrm{MPa}$ (abs);

(iii) pressure boundary condition at cold outlet with static pressure of $0.136 \mathrm{MPa}(\mathrm{abs})$;

(iv) zero gradient of temperature at both hot and cold outlet;

(v) no slip and adiabatic conditions at the wall.

\section{Energy Separation at Cryogenic Temperature}

The inlet stagnation temperature is considered to be $115 \mathrm{~K}$, which is marginally higher than the saturation temperature of air $(99.37 \mathrm{~K})$ at the inlet stagnation pressure. This is to ensure that air does not condense inside the tube during the process of energy separation. Values of air properties are obtained by using REFPROP software, version 7.0 [29]. The following values of the air properties (at $0.3 \mathrm{MPa}$ and $115 \mathrm{~K}$ ) are specified 
in the computational domain, $c_{p}=1071.1 \times \mathrm{Jkg}^{-1} \mathrm{~K}^{-1}, \mu=8.267$ $\times 10^{-6} \mathrm{kgm}^{-1} \mathrm{~s}^{-1}$, and $k=0.0115 \mathrm{Wm}^{-1} \mathrm{k}^{-1}$.

In the present study, thermodynamic properties are found to be predicted with good accuracy. The average deviation between the values of air density at each node of the computational domain at corresponding pressure and temperature obtained by the REFPROP software [29] and the value of air density computed by using perfect gas equation of state lies within $2 \%$. It should be noted that due to low inlet stagnation temperature $(115 \mathrm{~K})$, density throughout the tube is significantly higher than that in the normal temperature case. Density is found to vary from $4.4 \mathrm{kgm}^{-3}$ to $13.2 \mathrm{kgm}^{-3}$ inside the tube for inlet total pressure of $0.5422 \mathrm{MPa}$ and inlet total temperature of $115 \mathrm{~K}$. The specific heat of air varies with both pressure and temperature in the cryogenic range. However, the average deviation between the values of $c_{p}$ at each node of the computational domain at corresponding pressure and temperature obtained by using REFPROP [29] and the value of $c_{p}$ considered for computation $\left(c_{p}=1071.1 \mathrm{Jkg}^{-1} \mathrm{~K}^{-1}\right)$ lies within $2 \%$. The molecular viscosity and thermal conductivity also vary with both pressure and temperature at cryogenic temperature. However, CFD analysis of the VT shows that the turbulent viscosity $\left(\mu_{t}\right)$ and turbulent thermal conductivity $\left(k_{t}\right)$ are several times higher than the molecular viscosity and thermal conductivity (not reported here). Dutta et al. [17] also reported about high magnitude of $\mu_{t}$ inside the VT. Thus, the effects of the variation of molecular viscosity and thermal conductivity with pressure and temperature in the cryogenic range on the energy separation are negligible.

Figure 2 shows the pressure distribution in the VT. Variation of pressure with radius at various cross sections is presented. The section at $z=0.0008 \mathrm{~m}$ intersects the inlet boundary. This section is very close to the cold outlet boundary also. The section at $z=0.11 \mathrm{~m}$ is close to the hot outlet boundary. The figure shows that there is a pressure gradient in the radial direction throughout the tube with higher pressure near the periphery. The strongly swirling flow inside the VT causes the expansion of air from the wall to the axis. The pressure differential between the wall and the axis is maximum near the inlet $(0.27 \mathrm{MPa})$ and gradually decreases towards the hot outlet. The figure also shows that pressure is constant near $r=4.4 \mathrm{~mm}$ and decreases gradually from the inlet towards the hot outlet in the peripheral region. But in the core region, pressure decreases from the hot outlet towards the cold outlet. This indicates that air flows in the positive axial direction near the periphery, but in the core region, air flows in the negative axial direction. Figure 3, which shows the streamlines in the VT, clearly reveals the existence of return vortex flow in the core region.

Figure 4 shows the radial distribution of swirl velocity. Swirl velocity in the present case is found to be much smaller than that in the normal temperature case. For the VT operating with inlet total pressure of $0.5422 \mathrm{MPa}$ and inlet total temperature of $115 \mathrm{~K}$, the inlet swirl velocity is $137.9 \mathrm{~ms}^{-1}$ and the maximum swirl velocity inside the tube is $215.3 \mathrm{~ms}^{-1}$. Whereas, swirl velocity at inlet is $224.8 \mathrm{~ms}^{-1}$ and maximum swirl velocity is $343.5 \mathrm{~ms}^{-1}$ for the VT operating with inlet

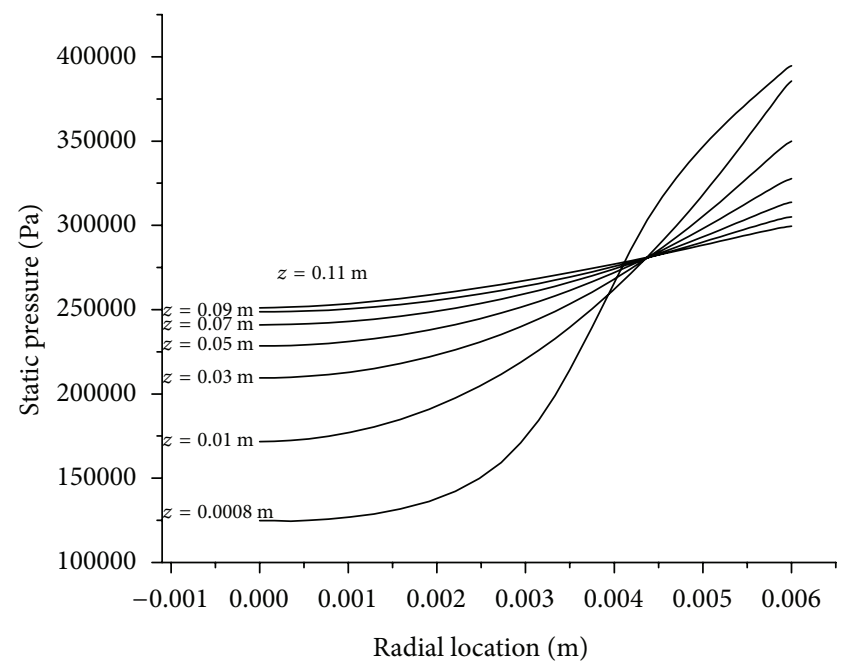

FIGURE 2: Radial distribution of static pressure $(\mathrm{Pa})$.

total pressure of $0.5422 \mathrm{MPa}$ and inlet total temperature of $300 \mathrm{~K}$ [17]. Similar trends are observed for other velocity components also. Smaller velocity components in cryogenic VT are attributed to the higher density of air. Figure 4 also shows that flow inside the VT is predominantly forced vortex flow. However, the curve corresponding to $z=0.0008 \mathrm{~m}$ indicates that there is a free vortex flow near the inlet. The reason can be found by examining Figure 2 . The curve corresponding to $z=0.0008 \mathrm{~m}$ suggests that air expands across the inlet nozzles of the VT from a total pressure of $0.5422 \mathrm{MPa}$ to a static pressure of $0.393 \mathrm{MPa}$ producing a high inlet swirl velocity $\left(137.9 \mathrm{~ms}^{-1}\right)$. Since the cold outlet is very close to the inlet, a portion of the flow expands further towards the cold outlet, thus producing maximum swirl velocity $\left(215.3 \mathrm{~ms}^{-1}\right)$ in this region.

Radial distribution of axial velocity is shown in Figure 5(a). Negative axial velocity in the core region confirms the existence of a return vortex flow as indicated in Figure 3. Figure 5(b) shows the contour plot of axial velocity $=0$. Across this contour surface of $w=0$, the flow changes its direction. The curve corresponding to $z=0.0008 \mathrm{~m}$ in Figure 5(a) shows a high magnitude of axial velocity $\left(-103 \mathrm{~ms}^{-1}\right)$ at the cold outlet region. This is due to a strong pressure gradient in the negative axial direction in the core region as indicated in Figure 2. Figure 6 shows the radial velocity distribution in the VT. Its value is $-35.5 \mathrm{~ms}^{-1}$ at the inlet. But, immediately after the inlet region, the radial velocity becomes insignificant.

Radial distribution of static temperature in the VT is shown in Figure 7. Static temperature is found to decrease marginally in radial direction, except near the inlet where the decrease is considerable. But, near the periphery, temperature is observed to increase sharply. The minimum static temperature is observed near the cold outlet region (94.5 K) owing to expansion of air from the inlet to the cold outlet. Static temperature is also found to increase in the axial direction. However, the static temperature difference between the wall and the axis decreases in the axial direction. 


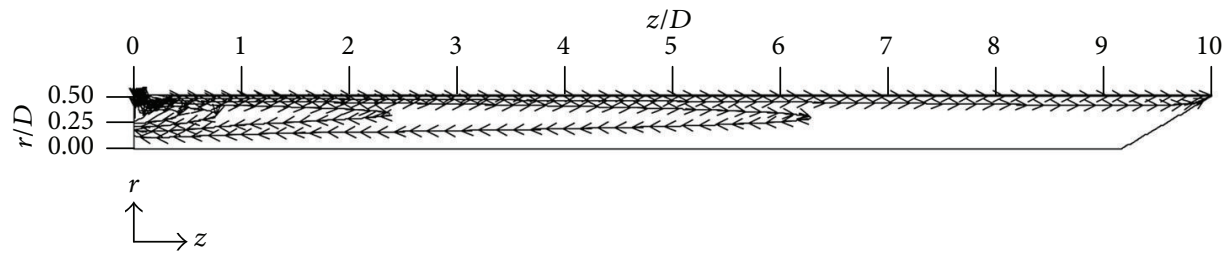

FIGURE 3: Streamlines in the vortex tube.

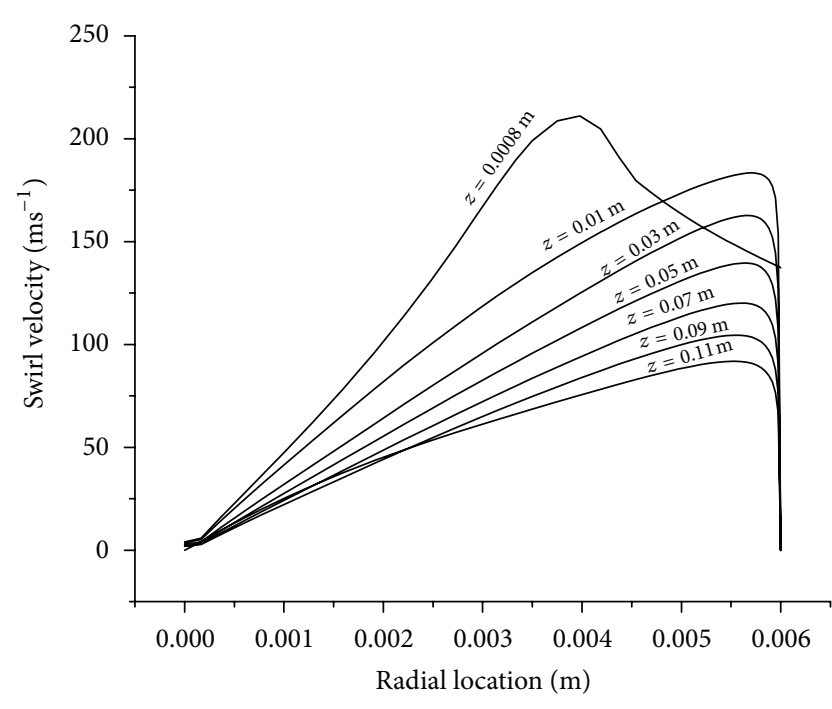

FIgURE 4: Radial distribution of swirl velocity $\left(\mathrm{ms}^{-1}\right)$.

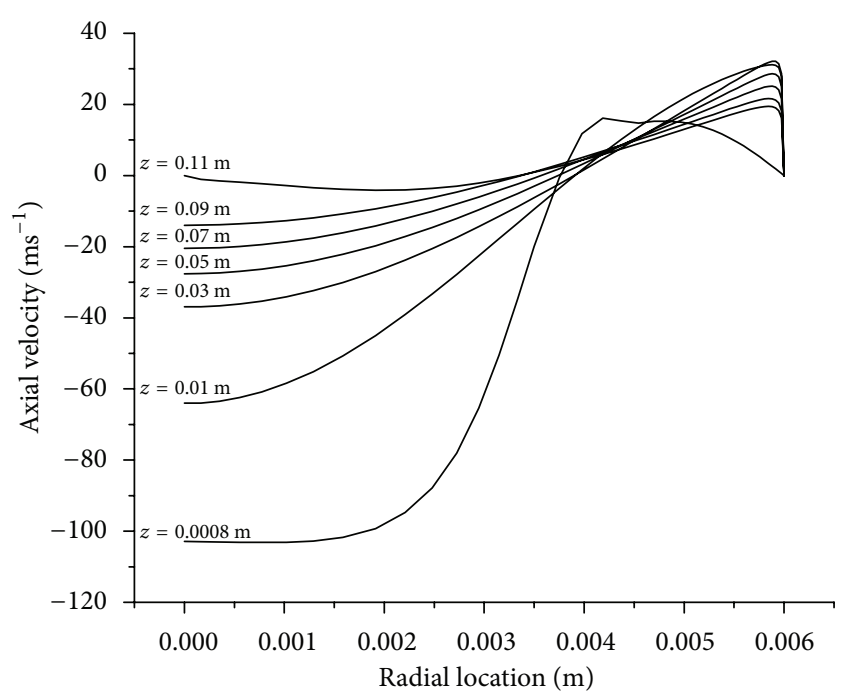

(a)

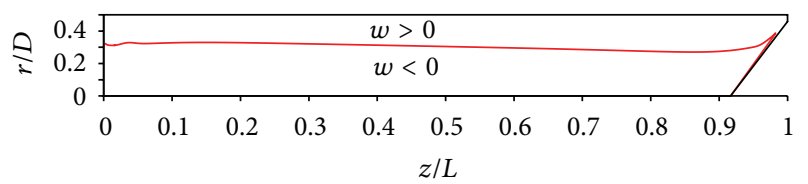

(b)

Figure 5: (a) Radial distribution of axial velocity $\left(\mathrm{ms}^{-1}\right)$. (b) Contour of axial velocity $=0$ inside the VT.

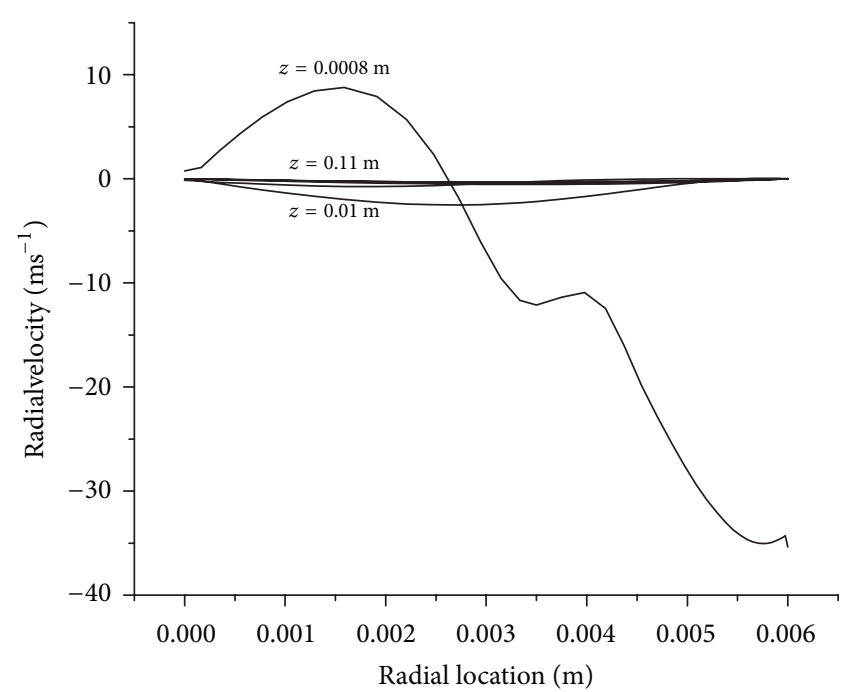

FIGURE 6: Radial distribution of radial velocity $\left(\mathrm{ms}^{-1}\right)$.

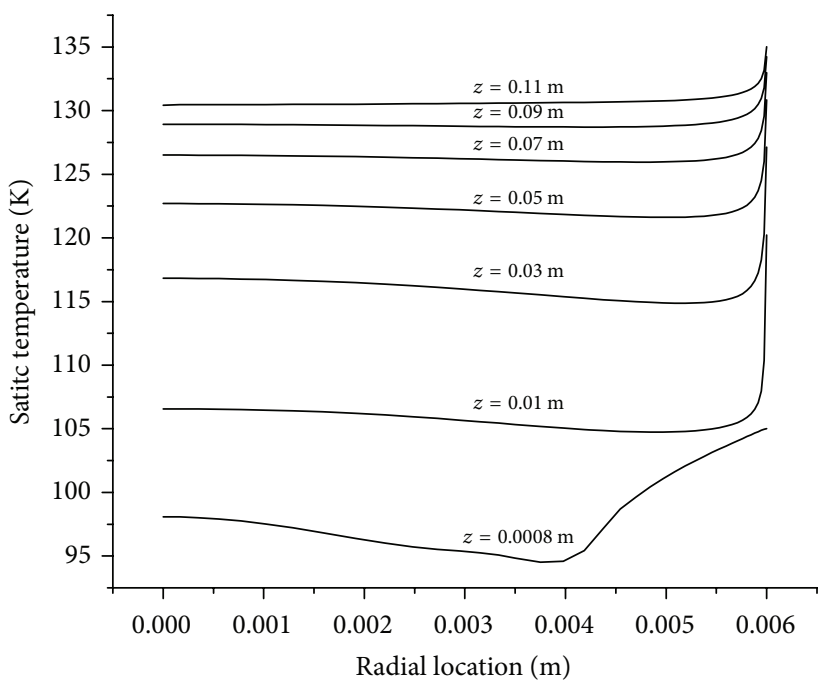

FIGURE 7: Radial distribution of static temperature (K).

Figure 8 shows the radial distribution of total temperature. The total temperature is found to increase gradually in both radial and axial directions. The energy separation phenomenon is clearly observed in this figure. The energy separation is attributed to transfer of viscous shear work from the inner fluid layers to the peripheral fluid layers as discussed later. Viscous shear work is transferred in the axial direction also, thus increasing the temperature in the 


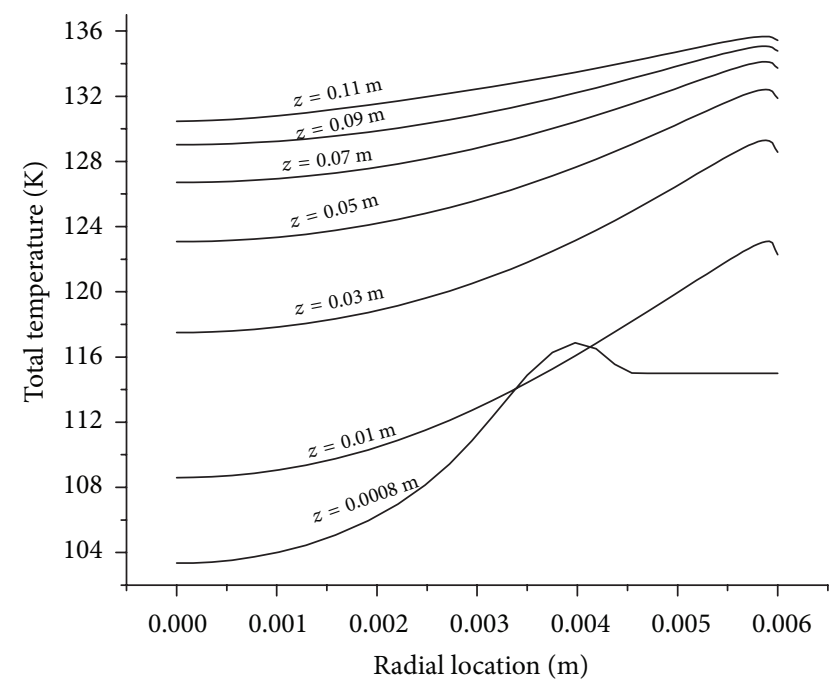

Figure 8: Radial distribution of total temperature (K).

axial direction. Near the wall, the total temperature is found to decrease, because velocity at the wall is zero due to no slip condition. The total temperatures at the hot and cold outlets are $135.3 \mathrm{~K}$ and $109.2 \mathrm{~K}$, respectively. On the other hand, CFD simulation of the same VT operating with same inlet and outlet pressures, but inlet stagnation temperature of $300 \mathrm{~K}$ shows that $T_{h}=349.2 \mathrm{~K}$ and $T_{c}=284.5 \mathrm{~K}$, respectively [17]. The value of $\left(T_{h}-T_{c}\right)$ in the VT at cryogenic temperature is significantly lower than that observed with normal atmospheric temperature air. This can be attributed to considerably lower total enthalpy $\left(107.61 \mathrm{kJkg}^{-1}\right)$ of air at $0.5422 \mathrm{MPa}$ and $115 \mathrm{~K}$ compared to total enthalpy of $299.54 \mathrm{kJkg}^{-1}$ of air at $0.5422 \mathrm{MPa}$ and $300 \mathrm{~K}$ (enthalpy of air obtained from REFPROP [29]).

To determine the rate of energy transfer between different fluid layers in the radial direction, control surfaces are considered at $r=0.1 \mathrm{~mm}, 0.5 \mathrm{~mm}$ to $5.5 \mathrm{~mm}$ in steps of $0.5 \mathrm{~mm}$, and $5.9 \mathrm{~mm}$. The rate of heat transfer $(\delta \dot{Q})$ and work transfer due to viscous shear stress $(\delta \dot{W})$ normal to the control surfaces are calculated across a differential length of the control surface $(d z)$ and then integrated over the whole control surface to obtain the overall rate of heat and work transfer across the surface. The rate of sensible heat transfer normal to the control surfaces per unit length is given by

$$
\frac{\delta \dot{Q}}{d z}=-2 \pi r k_{\mathrm{eff}} \frac{\partial T}{\partial r} .
$$

The term " $T$ " on the right side of (1) indicates "static temperature". $k_{\text {eff }}=k+\left(c_{p} \mu_{t} / \mathrm{Pr}_{t}\right)$ is the effective thermal conductivity, where $\operatorname{Pr}_{t}=0.85$ is the turbulent Prandtl number.

The rate of work transfer due to tangential shear stress normal to the control surfaces per unit length is given by

$$
\frac{\delta \dot{W}_{\theta}}{d z}=-2 \pi r \mu_{\mathrm{eff}} u\left(\frac{\partial u}{\partial r}-\frac{u}{r}\right) .
$$

Here, $\mu_{\text {eff }}=\mu+\mu_{t}$ is the effective viscosity.
The rate of work transfer due to axial shear stress normal to the control surfaces per unit length is given by

$$
\frac{\delta \dot{W}_{z}}{d z}=-2 \pi r \mu_{\mathrm{eff}} w \frac{\partial w}{\partial r}
$$

Figures 9(a) and 9(b) show the rate of energy transfer per unit length across the control surfaces at $r=2 \mathrm{~mm}$ and $5 \mathrm{~mm}$. Figure 9(a) shows that at $r=2 \mathrm{~mm}$, both tangential shear work and axial shear work are transferred in the positive radial direction (i.e., from inner fluid layer to outer fluid layer). Thus, the outer fluid layer gains energy and its total temperature increases. It is also found that tangential shear work is much smaller compared to axial shear work despite higher magnitude of swirl velocity than axial velocity at $r=$ $2 \mathrm{~mm}$. This is due to the fact that the value of $((\partial u / \partial r)-$ $(u / r))$ is considerably lower than $(\partial w / \partial r)$. Sensible heat is also transferred from the inner to outer fluid layer across the control surface due to temperature gradient.

Figure 9(b) shows that at $r=5 \mathrm{~mm}$, only the tangential shear work is transferred from inner to outer fluid layer, whereas axial shear work and sensible heat are transferred from outer to inner fluid layer. Thus, the magnitude of energy separation is reduced to some extent due to transfer of axial shear work and heat in the negative radial direction near the periphery. Here, sensible heat is transferred from outer to inner fluid layer due to higher static temperature near the periphery as shown in Figure 7. Positive axial shear work at $r=2 \mathrm{~mm}$ and negative axial shear work at $r=5 \mathrm{~mm}$ are due to the opposite direction of flows at the peripheral and core region, as shown in Figure 3.

Figures 9(a) and 9(b) also show that the magnitude of tangential and axial shear work and sensible heat transfer are significantly higher within the first $2 \mathrm{~cm}$ length of the rube. This is because of higher values of effective viscosity and effective thermal conductivity in this region. Maximum effective viscosity of $0.1379 \mathrm{kgm}^{-1} \mathrm{~s}^{-1}$ and maximum effective thermal conductivity of $163.28 \mathrm{Wm}^{-1} \mathrm{~K}^{-1}$ are observed in this region. Also, the absolute values of the rate of heat transfer and work transfer are much higher at $r=5 \mathrm{~mm}$ than those at $r=2 \mathrm{~mm}$.

Equations (1), (2), and (3) are integrated over the length of the VT for each radial location mentioned earlier to calculate the total rate of work and sensible heat transfer across each control surface. These values are plotted in Figure 10. This figure clearly reveals the mechanism of energy separation. Transfer of tangential shear work from the inner fluid layers to the outer fluid layers is found to be the cause of energy separation in the VT. It may be noted that through numerical investigation of VT operating with air at normal atmospheric temperature [30] it has been found that angular velocity decreases radially outwards in a VT. This gradient of angular velocity leads to the transfer of angular momentum from fast moving inner fluid layers to the slow moving peripheral fluid layers by means of viscous shear work. The theory of viscous shear work as the cause of energy separation was proposed by Hilsch [2] and later supported by numerous experimental, theoretical and numerical studies (see [9, 30-34], etc.). All of 


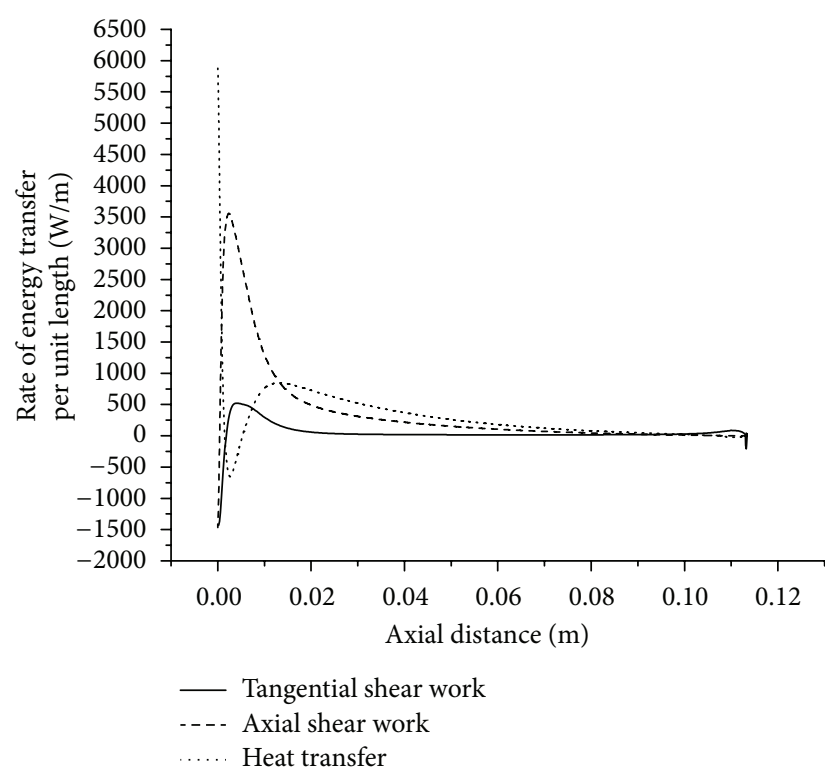

(a)

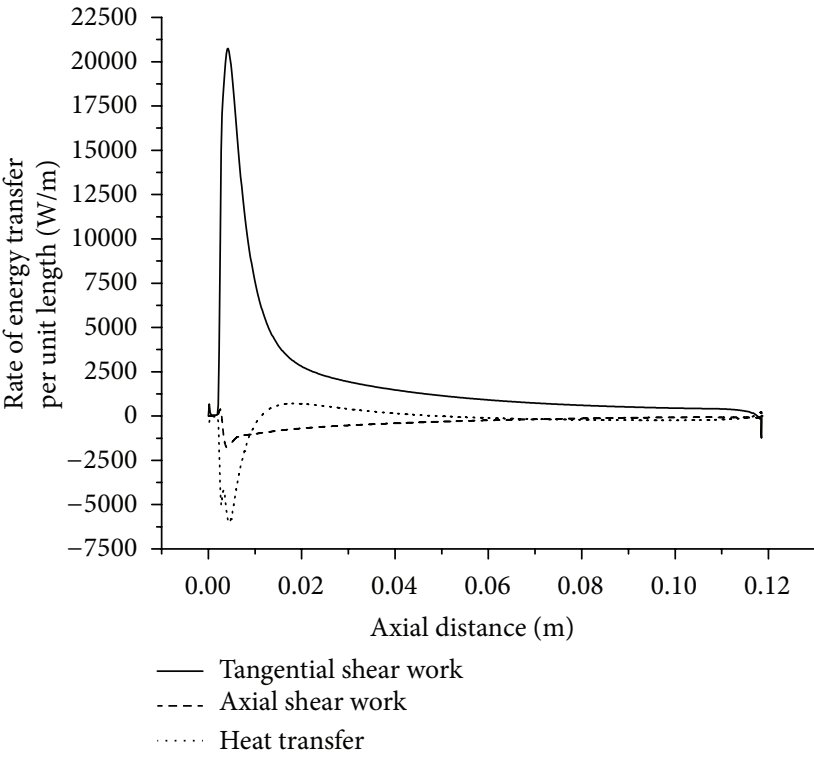

(b)

Figure 9: Rate of energy transfer per unit length $\left(\mathrm{Wm}^{-1}\right)$ across the control surface at (a) $r=2 \mathrm{~mm}$, (b) $r=5 \mathrm{~mm}$.

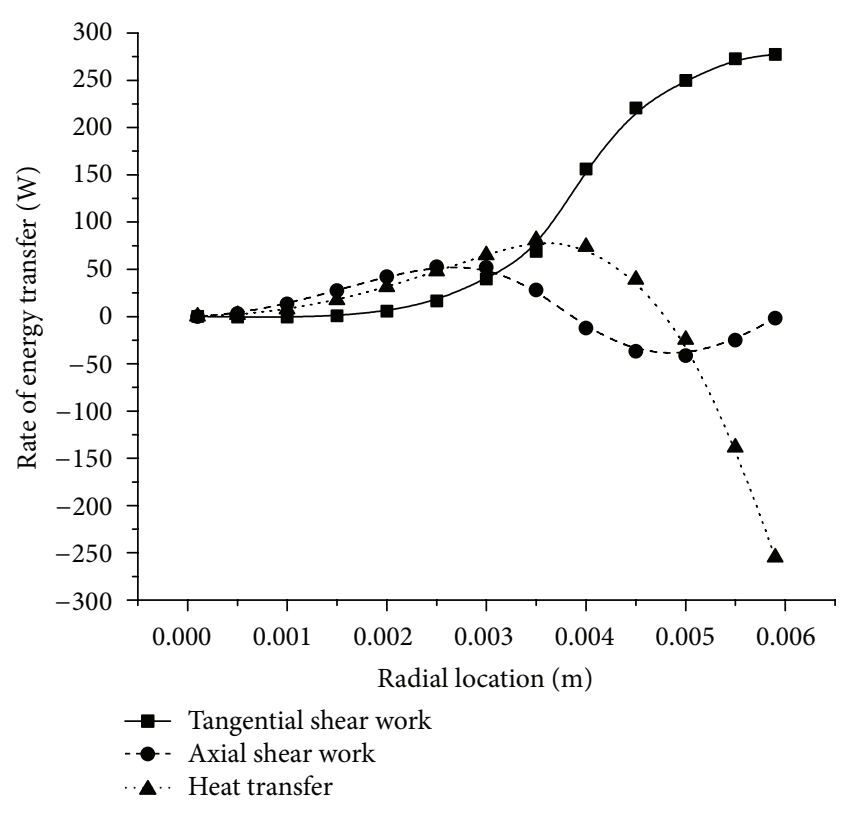

FIGURE 10: Total energy transfer rate $(\mathrm{W})$ across the control surfaces at various radial locations.

these studies emphasize on the great influence of turbulence on energy separation.

From Figure 10, it is observed that as the swirl velocity increases in the radial direction, transfer of tangential shear work also increases. Axial shear work increases in the radial direction in the core region but reduces in the region at $r=3 \mathrm{~mm}$ to $r=3.9 \mathrm{~mm}$ due to lower axial velocity. Above $r=3.9 \mathrm{~mm}$, direction of flow becomes opposite to that in the core region, thus producing negative axial shear work. This reduces the magnitude of energy separation. Transfer of sensible heat also increases with radial distance in the core region. However, due to higher static temperature near the wall, as observed in Figure 7, sensible heat is transferred from outer to inner fluid layers at the peripheral region. This phenomenon reduces the overall energy separation to some extent.

To determine the rate of energy transfer between different fluid layers in the axial direction, control surfaces are considered at the cross sections at $z=0.8 \mathrm{~mm}, 2 \mathrm{~mm}, 4 \mathrm{~mm}, 6 \mathrm{~mm}$, $8 \mathrm{~mm}, 10 \mathrm{~mm}$ to $110 \mathrm{~mm}$ in steps of $10 \mathrm{~mm}, 119.5 \mathrm{~mm}$, and $120 \mathrm{~mm}$. The rate of heat transfer $(\delta \dot{Q})$ and work transfer due to viscous shear stress $(\delta \dot{W})$ normal to the control surfaces are calculated across a differential length of the control surface $(d r)$ and then integrated over the whole control surface to obtain the overall rate of heat and work transfer across that surface. The rate of transfer of sensible heat per unit length is calculated by

$$
\frac{\delta \dot{Q}}{d r}=-2 \pi r k_{\mathrm{eff}} \frac{\partial T}{\partial z}
$$

The term " $T$ " on the right hand side of (4) indicates "static temperature."

The rate of work transfer per unit length due to viscous shear in the tangential direction is given by

$$
\frac{\delta \dot{W}_{\theta}}{d r}=-2 \pi r \mu_{\mathrm{eff}} u \frac{\partial u}{\partial z}
$$

The rate of work transfer per unit length due to viscous shear in the radial direction is given by

$$
\frac{\delta \dot{W}_{r}}{d r}=-2 \pi r \mu_{\mathrm{eff}} v \frac{\partial v}{\partial z} .
$$




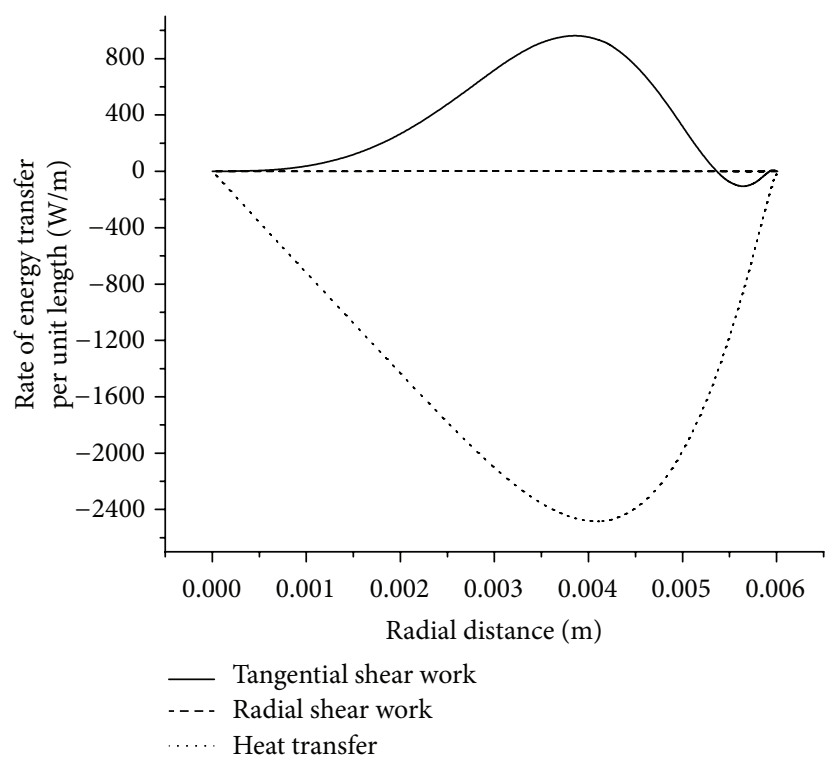

(a)

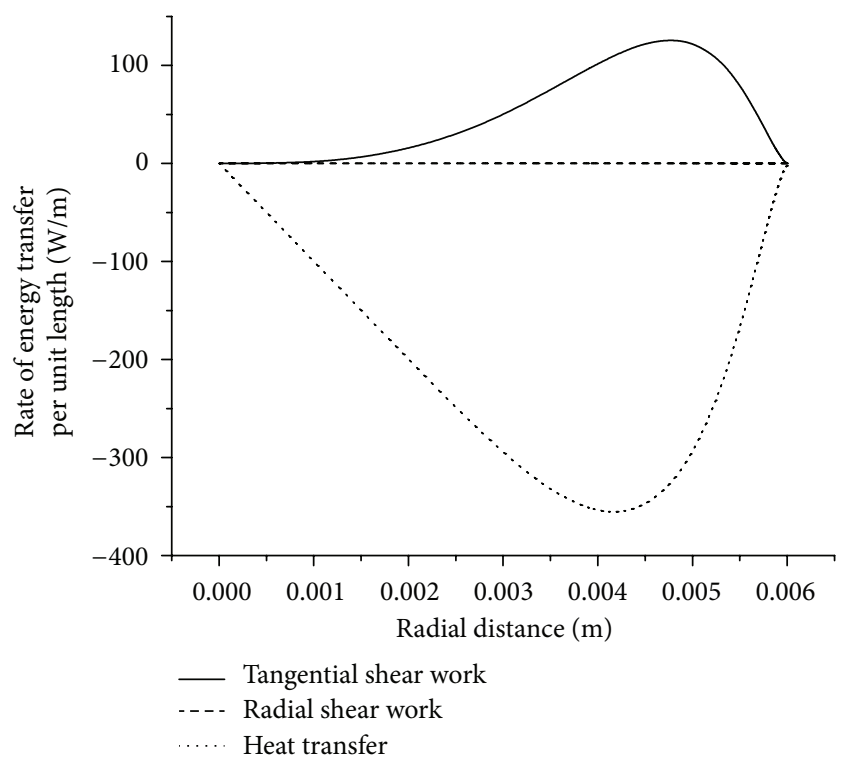

(b)

FIGURE 11: Rate of energy transfer per unit length $\left(\mathrm{Wm}^{-1}\right.$ ) across the control surfaces at (a) $z=10 \mathrm{~mm}$, (b) $z=60 \mathrm{~mm}$.

Figures 11(a) and 11(b) show the rate of energy transfer per unit length across the control surfaces at $z=10 \mathrm{~mm}$ and $60 \mathrm{~mm}$. Both figures show that the work transfer due to radial shear stress is negligible. It is also observed that tangential shear work is transferred in positive axial direction, whereas heat transfer occurs in the opposite direction. However, magnitude of energy transfer at $z=60 \mathrm{~mm}$ is much lower than that at $z=10 \mathrm{~mm}$. This is due to lower values of $(\partial T / \partial z), k_{\mathrm{eff}}$, $(\partial u / \partial z), u$, and $\mu_{\text {eff }}$ at $z=60 \mathrm{~mm}$. Also, comparing Figures 11(a) and 11(b) with Figures 9(a) and 9(b), magnitude of the energy transfer per unit length in radial direction is found to be higher than that in the axial direction.

Equations (4), (5), and (6) are integrated over the cross section for all the axial locations mentioned above to compute the total rate of work and heat transfer across each cross section. These values are presented in Figure 12. This figure shows that work transfer in axial direction due to radial shear stress is very small throughout the VT. The tangential shear work always takes place in positive axial direction, thus enhancing the energy separation between hot and cold outlets. On the other hand, transfer of sensible heat always takes place in the negative axial direction, thus reducing the overall energy separation. Magnitude of both, work and heat transfers, is maximum near the inlet region. Since magnitude of work transfer is lower than the magnitude of heat transfer in the axial direction, net energy transfer in this direction reduces the energy separation.

\section{Parametric Sensitivity Analysis}

4.1. Variation with Hot Outlet Mass Fraction. The hot outlet mass fraction, $\xi$, is varied by changing the hot outlet opening, e-f. Variation of $T_{h}$ and $T_{c}$ with $\xi$ is shown in Figure 13. It shows that both $T_{h}$ and $T_{c}$ decrease as $\xi$ increases. This

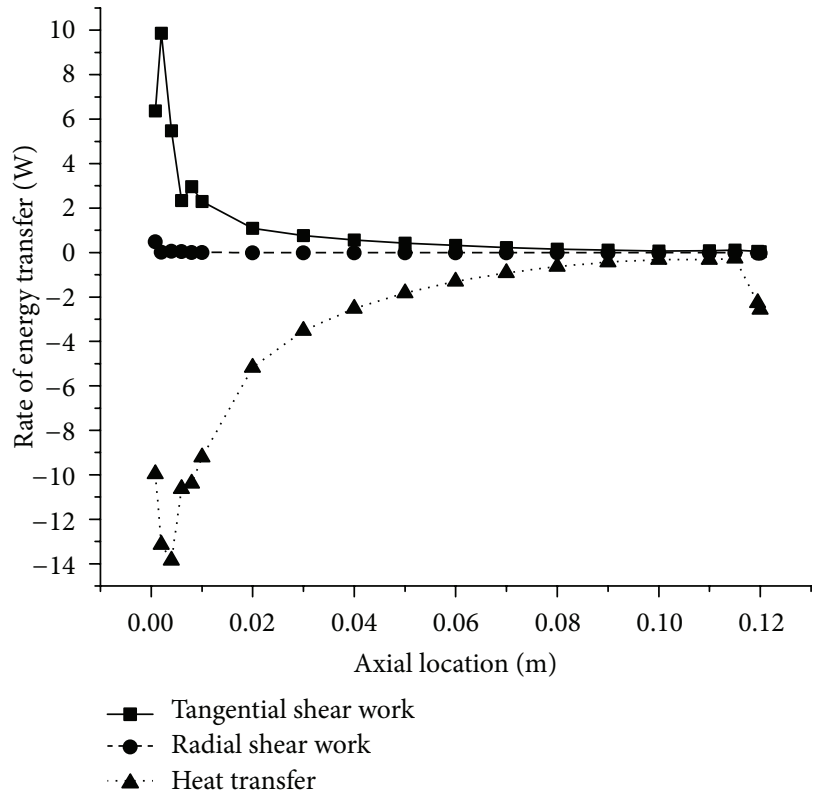

FIGURE 12: Total energy transfer rate (W) across the control surfaces at various axial locations.

behavior is similar to that reported for VTs operating with normal atmospheric temperature air [17]. To determine the effect of $\xi$ on the rate of energy transfer between different fluid layers in the radial direction, control surfaces are considered at different radial locations mentioned in Section 3. Equations (1), (2), and (3) are integrated over the length of the VT at all radial locations to calculate the total rate of work and sensible heat transfer across each control surface. These values are plotted in Figure 14. It shows that the transfer of tangential shear work from the inner fluid layers to the outer 


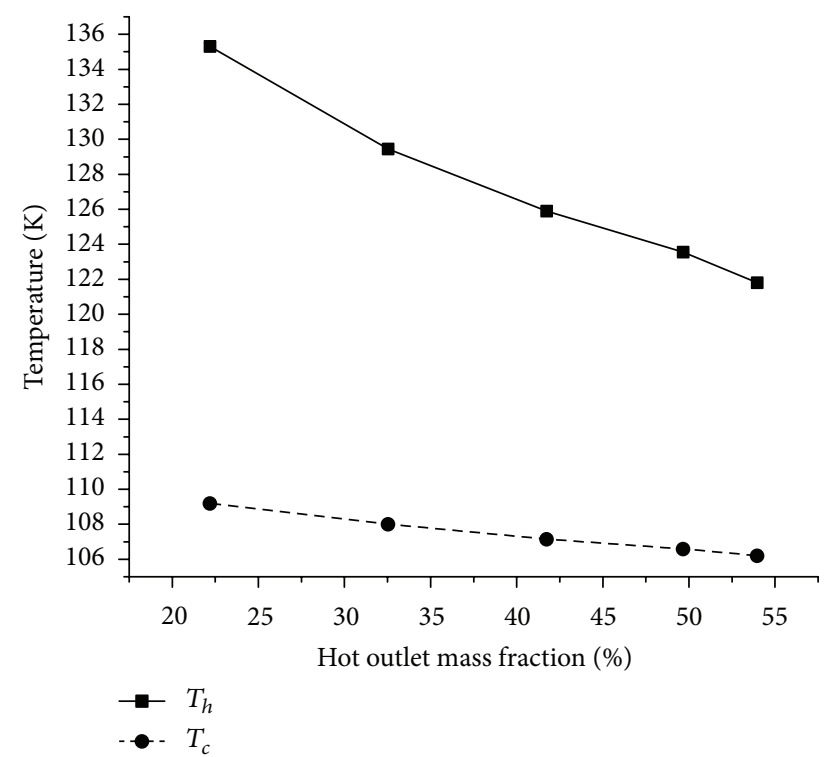

FIGURE 13: Variation of hot and cold outlet temperature (K) with hot outlet mass fraction.

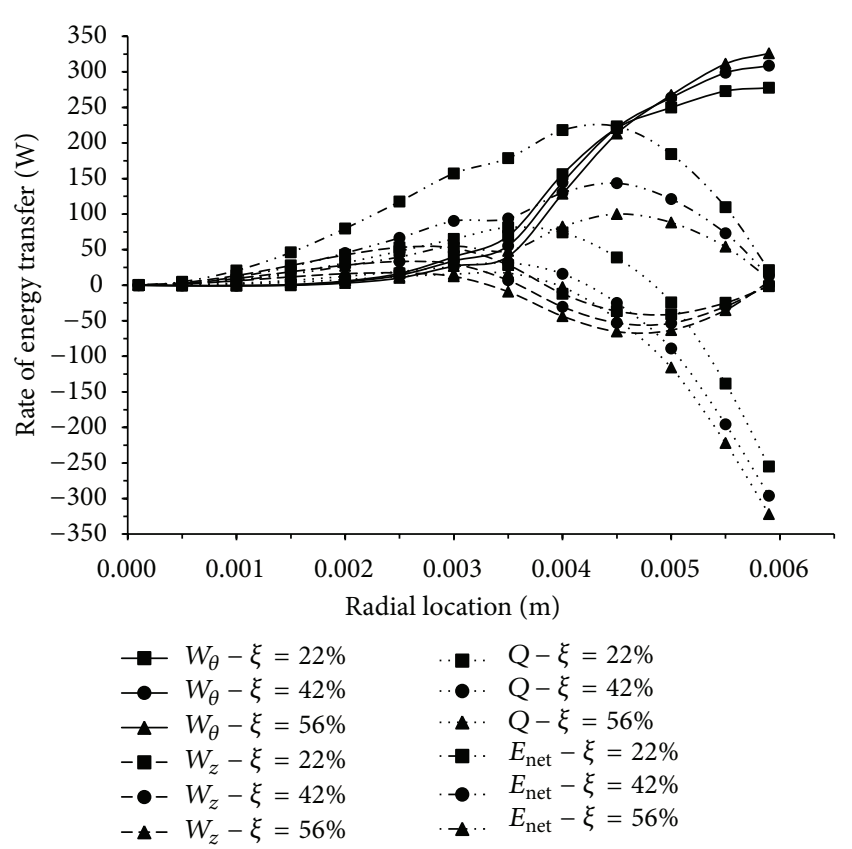

FIGURE 14: Rate of energy transfer (W) across the control surfaces at various radial locations for different hot outlet mass fraction.

fluid layers increases marginally near the periphery of the VT for higher values of $\xi(\xi=56 \%)$, while the transfer of axial shear work and sensible heat from the inner fluid layers to the outer fluid layers decrease significantly with increase in $\xi$. The net energy transfer, $\dot{E}_{\text {net }}=\dot{Q}+\dot{W}_{\theta}+\dot{W}_{z}$, from the inner fluid layers to the outer fluid layers is found to decrease considerably for higher values of $\xi$. Thus, energy separation decreases with increase in $\xi$ as observed in Figure 13.

4.2. Variation with Inlet Pressure. The effect of variation of the inlet pressure on energy separation at cryogenic temperature

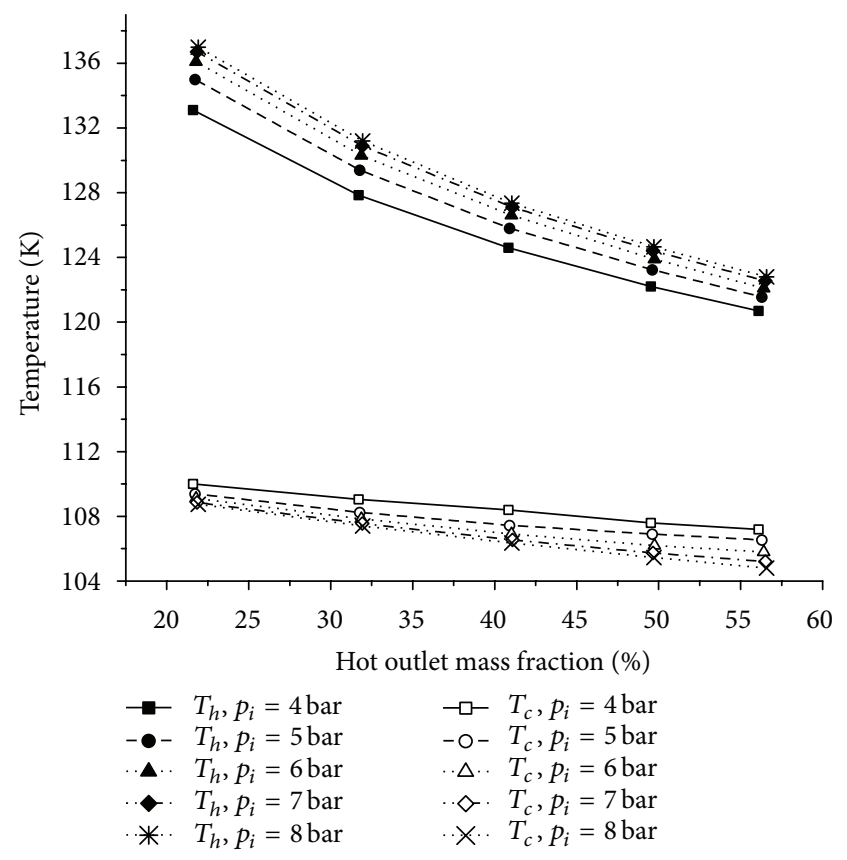

FIGURE 15: Variation of hot and cold outlet temperature (K) for different inlet pressure.

has been investigated by varying the inlet stagnation pressure from $0.4 \mathrm{MPa}$ to $0.8 \mathrm{MPa}$. The stagnation temperature is maintained at $115 \mathrm{~K}$. Variation of $T_{h}$ and $T_{c}$ with the inlet pressure is shown in Figure 15. It is observed that $T_{h}$ increases and $T_{c}$ decreases with increase in inlet pressure. Thus, the energy separation increases with inlet pressure. However, for the VT considered, the increase in temperature separation is found to be small for inlet pressure higher than $0.7 \mathrm{MPa}$.

To determine the effect of variation of the inlet pressure on the rate of energy transfer between different fluid layers in the radial direction, control surfaces are considered at different radial locations mentioned in Section 3. The rates of heat and work transfer normal to the control surfaces for $\xi=41 \%$ is calculated as before. These values are plotted in Figure 16. It shows that the transfer of tangential shear work from the inner fluid layers to the outer fluid layers increases significantly for higher inlet pressure. This happens due to increased swirl velocity in the VT caused by increased inlet pressure. Transfer of axial shear work from the inner fluid layers to the outer fluid layers increases in the core region with inlet pressure, while it decreases near the peripheral region of the tube. Transfer of sensible heat from the inner fluid layers to the outer fluid layers decreases near the peripheral region with inlet pressure. The net energy transfer from the inner fluid layers to the outer fluid layers increases considerably with increase in inlet pressure. This is manifested by higher magnitude of energy separation with increase in inlet pressure as shown in Figure 15.

4.3. Variation with Length to Diameter Ratio $(L / D)$ of the VT. $L / D$ is varied from 7.5 to 15 in steps of 2.5 to determine the influence of $L / D$ on the energy separation. The variations 


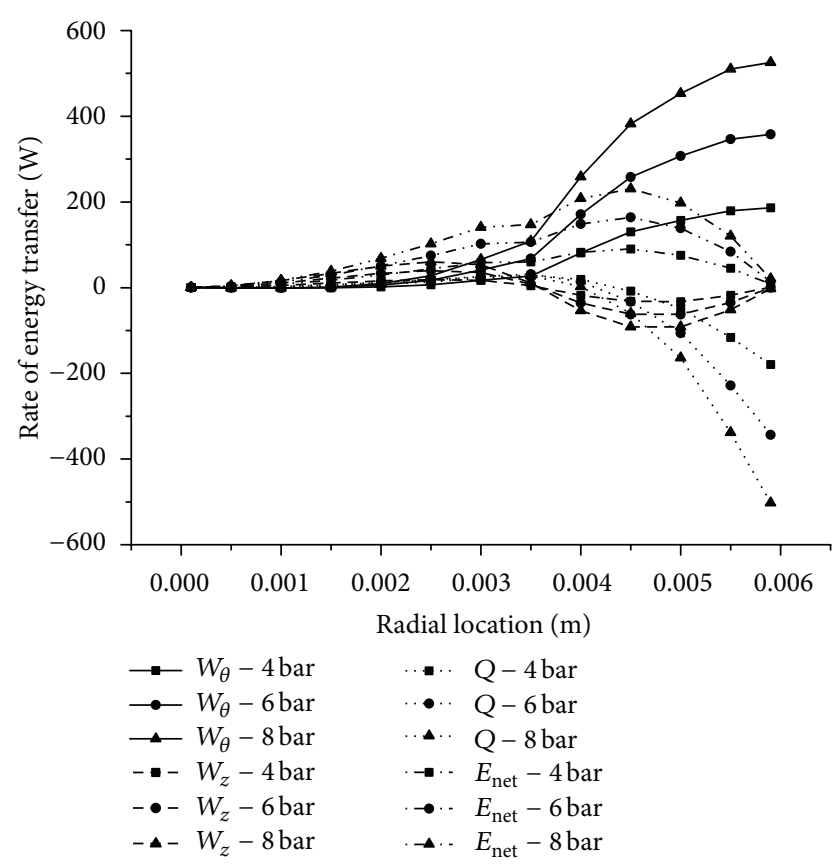

FIGURE 16: Rate of energy transfer (W) across the control surfaces at various radial locations for different inlet pressure.

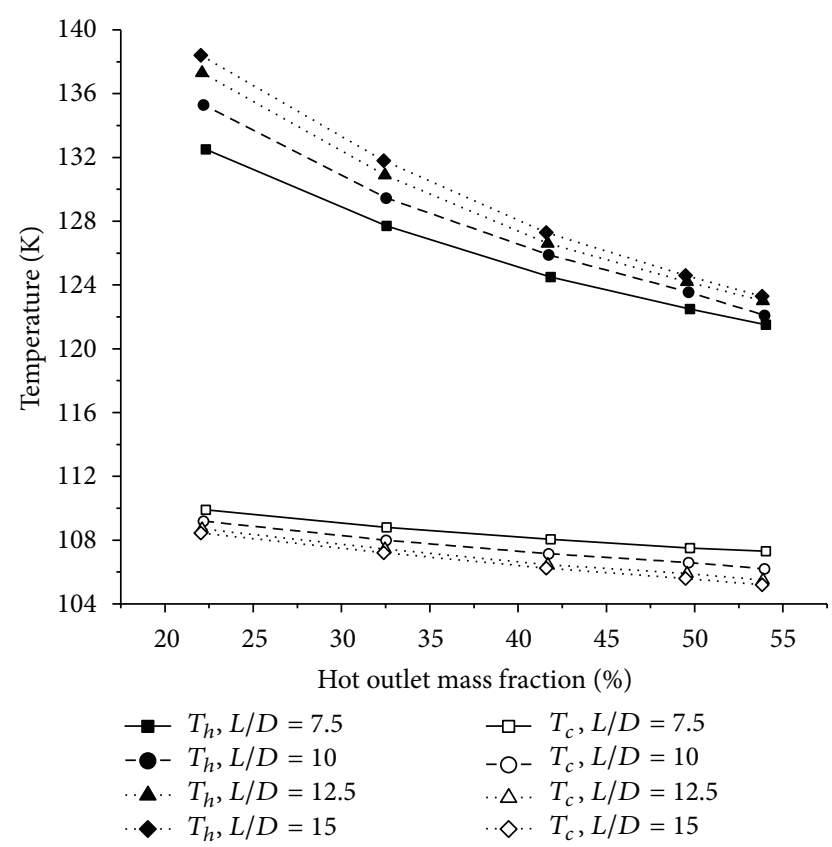

FIGURE 17: Variation of hot and cold outlet temperature (K) for different $L / D$.

of $T_{h}$ and $T_{c}$ with $\xi$ for different $L / D$ values are shown in Figure 17. It is observed that $T_{h}$ increases and $T_{c}$ decreases with the increase in $L / D$. In other words, magnitude of temperature separation increases with $L / D$. However, the increase in the magnitude of temperature separation reduces with successive increase in $L / D$. Beyond $L / D=12.5$, the increase in temperature separation becomes insignificant.
From the CFD analysis of the VT operating with normal atmospheric temperature air, it has been found that there is a critical $L / D$ of the VT above which the starting point of the return vortex flow lies within the tube. Increase in temperature separation is not observed by increasing the $L / D$ of the tube beyond this critical value. It may be noted that Behera et al. [10] found the critical $L / D$ to be 30 for VTs operating with air at normal atmospheric temperature. Due to lower magnitude of velocity inside the cryogenic VT, the starting point of the return vortex flow lies within the VT for $L / D \geq 12.5$. Thus, significant improvement in temperature separation is not observed for $L / D \geq 12.5$ for VTs operating at cryogenic temperature.

4.4. Variation with the Location of Inlet. Figure 1 shows the inlet located at the end of the VT near the cold outlet. CFD analysis is carried out to investigate whether temperature separation is influenced if the inlet is gradually shifted from its original location. The modified working geometry of the $\mathrm{VT}$ is shown in Figure 18. The distance $\mathrm{c}-\mathrm{c}^{\prime}$ between the inlet and the cold outlet, denoted by $\zeta$, is allowed to vary between $0 \mathrm{~mm}$ and $18 \mathrm{~mm}$ in steps of $6 \mathrm{~mm}$. Variations of $T_{h}$ and $T_{c}$ with the inlet locations are plotted in Figure 19. It is observed that $T_{h}$ decreases and $T_{c}$ increases as $\zeta$ increases. Thus, the energy separation decreases as the inlet is shifted away from the cold outlet.

The rate of energy transfer between different fluid layers in the radial direction across a control surface at $r=5 \mathrm{~mm}$ is analyzed to determine the effect of inlet location. Values of $\dot{Q}, \dot{W}_{\theta}$, and $\dot{W}_{z}$ normal to the control surface per unit length are calculated by using (1)-(3) at $\xi=41 \%$ for $\zeta=$ $0 \mathrm{~mm}$ and $\zeta=18 \mathrm{~mm}$. These values are plotted in Figures 20 (a) and 20(b). Also, profiles of total temperature for $\zeta=$ $0 \mathrm{~mm}$ and $\zeta=18 \mathrm{~mm}$ are shown in Figures 21(a) and 21(b). It is found that major portion of the tangential shear work transfer from the inner fluid layers to the outer fluid layers takes place between the cold outlet and inlet $\left(c-c^{\prime}\right)$ as the inlet is shifted from the cold outlet. Temperature near the peripheral region between $c-c^{\prime}$ increases as a result of this work transfer. However, this tangential shear work transfer does not contribute to increasing the hot outlet temperature. Thus, $T_{h}$ decreases with the increase in $\zeta$. Also, higher amount of sensible heat transfer from the outer fluid layers to inner fluid layers in the region $c-c^{\prime}$ increases $T_{c}$. Thus, $T_{c}$ increases as $\zeta$ increases. Hence, the inlet should be located nearest to the cold outlet for higher temperature separation.

4.5. Variation For Different Working Fluids. To investigate the influence of different working fluids on energy separation, nitrogen, oxygen, hydrogen and argon are respectively chosen as working fluids. Table 1 presents the values of the fluid properties (at $0.3 \mathrm{MPa}$ and $115 \mathrm{~K}$ ) obtained from the REFPROP software [29], which are specified for computation.

All working fluids are considered to follow the perfect gas equation of state. Variation of $T_{h}$ and $T_{c}$ for each of the above mentioned gases as working fluid is shown in Figure 22. $T_{h}$ and $T_{c}$ obtained for air, nitrogen, and oxygen are found to be almost the same. The values of $T_{h}$ obtained with hydrogen 


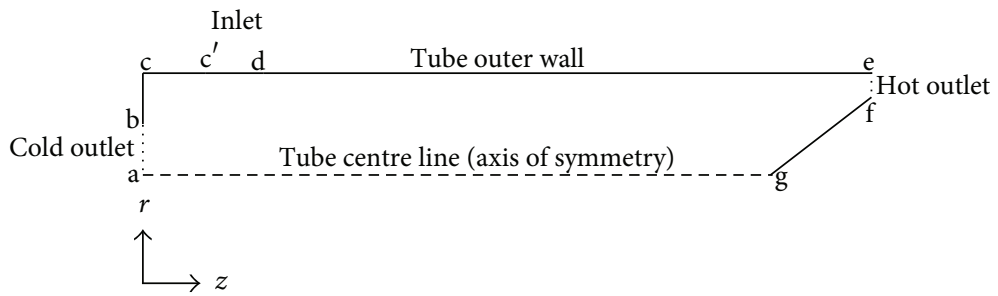

FIGURE 18: Modified working geometry of the vortex tube for different inlet locations.

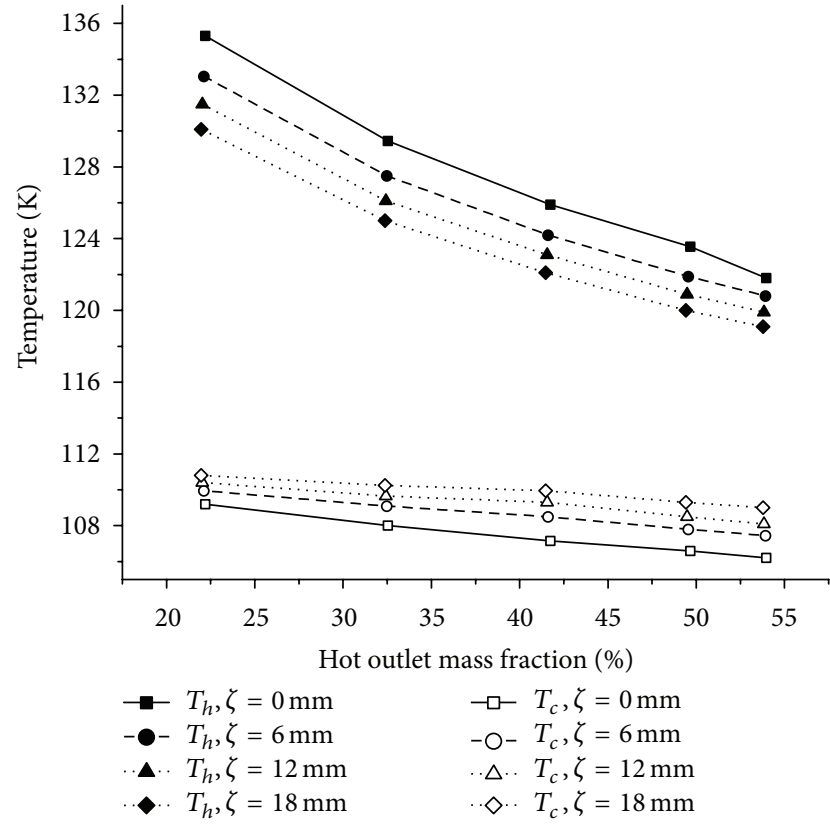

FIgURE 19: Variation of hot and cold outlet temperature (K) with inlet location.

TABLE 1: Properties of different working fluids at $0.3 \mathrm{MPa}$ and $115 \mathrm{~K}$.

\begin{tabular}{lcccc}
\hline $\begin{array}{l}\text { Working } \\
\text { fluid }\end{array}$ & $\begin{array}{c}c_{p} \\
\left(\mathrm{Jkg}^{-1} \mathrm{~K}^{-1}\right)\end{array}$ & $\begin{array}{c}\mu \\
\left(\mathrm{kgm}^{-1} \mathrm{~s}^{-1}\right)\end{array}$ & $\begin{array}{c}k \\
\left(\mathrm{Wm}^{-1} \mathrm{k}^{-1}\right)\end{array}$ & $\begin{array}{c}M \\
\left(\mathrm{kgkmol}^{-1}\right)\end{array}$ \\
\hline Air & 1071.1 & $8.267 \times 10^{-6}$ & 0.0115 & 28.96 \\
$\mathrm{~N}_{2}$ & 1106 & $8.026 \times 10^{-6}$ & 0.01134 & 28.013 \\
$\mathrm{O}_{2}$ & 976.5 & $9.0533 \times 10^{-6}$ & 0.01092 & 32 \\
$\mathrm{H}_{2}$ & 11710 & $4.636 \times 10^{-6}$ & 0.07863 & 2.016 \\
$\mathrm{Ar}$ & 575.26 & $9.547 \times 10^{-6}$ & 0.0075 & 39.95 \\
\hline
\end{tabular}

and argon are significantly higher than those obtained with the other gases. Also, the values of $T_{c}$ obtained with hydrogen and argon are significantly lower than those obtained with the other gases. The simulated results establish that argon is the most efficient working fluid in terms of energy separation. This is attributed to the lower value of $c_{p} M$ of argon. The factor $c_{p} M$ is calculated for all the gases considered and the values are $\left[c_{p} M\right]_{\mathrm{Air}}=31019.1 \mathrm{Jkmol}^{-1} \mathrm{~K}^{-1},\left[c_{p} M\right]_{\mathrm{N} 2}=$ $30982.4 \mathrm{Jkmol}^{-1} \mathrm{~K}^{-1},\left[c_{p} M\right]_{\mathrm{O} 2}=31248 \mathrm{Jkmol}^{-1} \mathrm{~K}^{-1},\left[c_{p} M\right]_{\mathrm{H} 2}$ $=23607.4 \mathrm{Jkmol}^{-1} \mathrm{~K}^{-1}$, and $\left[c_{p} M\right]_{\mathrm{Ar}}=22981.6 \mathrm{Jkmol}^{-1} \mathrm{~K}^{-1}$.
Figure 22 clearly indicates that the gas having higher value of the factor $c_{p} M$ produces lower temperature separation. The values of $c_{p} M$ for air, nitrogen, and oxygen are very close thus, the temperature separation with these gases is nearly identical. Similarly, temperature separations observed with hydrogen and argon are very close due to the same reason. Argon, which has the lowest value of $c_{p} M$, produces the maximum temperature separation.

\section{Conclusions}

In the present study, a CFD analysis is carried out to simulate the energy separation phenomenon in VT with gaseous air at cryogenic temperature as working fluid. From the study the following conclusions are obtained.

(i) Magnitude of velocity components in the cryogenic VT is found to be significantly smaller compared to that at normal atmospheric temperature due to higher density of air at cryogenic temperature.

(ii) Transfer of tangential shear work from inner to outer fluid layers is the driving force for energy separation in the VT. Similar observation was made by several researchers in case of VT operating at normal atmospheric temperature. Energy separation at cryogenic temperature is found to be considerably less than that at normal atmospheric temperature due to lower values of inlet enthalpy and velocity.

(iii) Transfer of tangential shear work in axial direction enhances the energy separation. However, heat transfer and transfer of axial shear work from outer to inner fluid layers near the periphery of VT deteriorate the energy separation. Also, heat transfer in negative axial direction reduces the energy separation. However, work transfer due to radial shear stress is very small throughout the VT.

(iv) Energy separation decreases with increase in hot outlet mass fraction because of the fact that transfer of axial shear work and sensible heat from the inner fluid layers to outer fluid layers decreases significantly with increase in hot outlet mass fraction.

(v) Energy separation increases with increase in inlet pressure. This is due to the fact that higher swirl velocity is generated at higher inlet pressure, and subsequently transfer of tangential shear work from the inner fluid layers to the outer fluid layers increases 


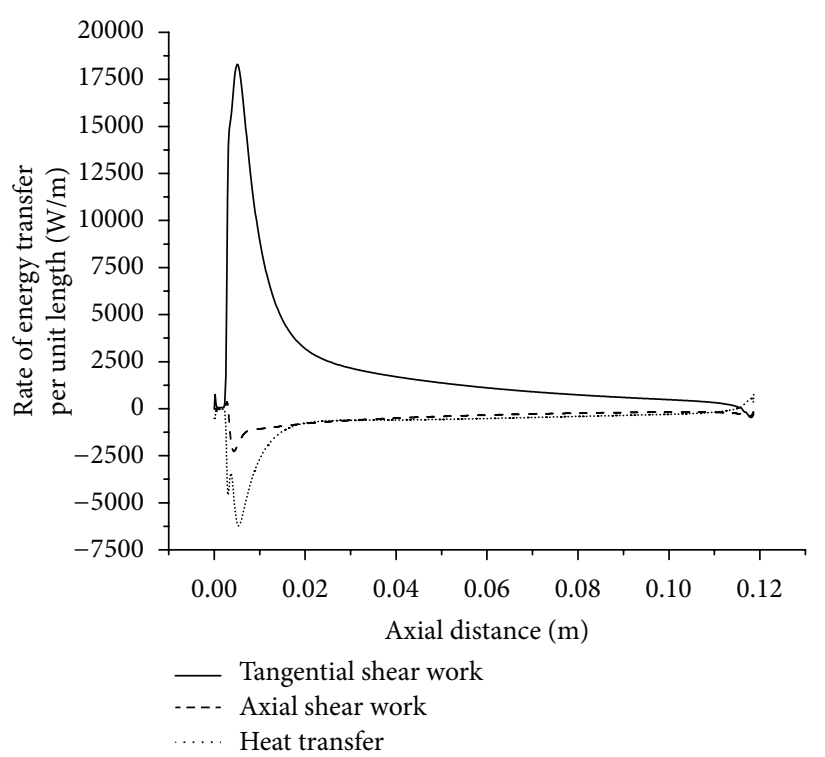

(a)

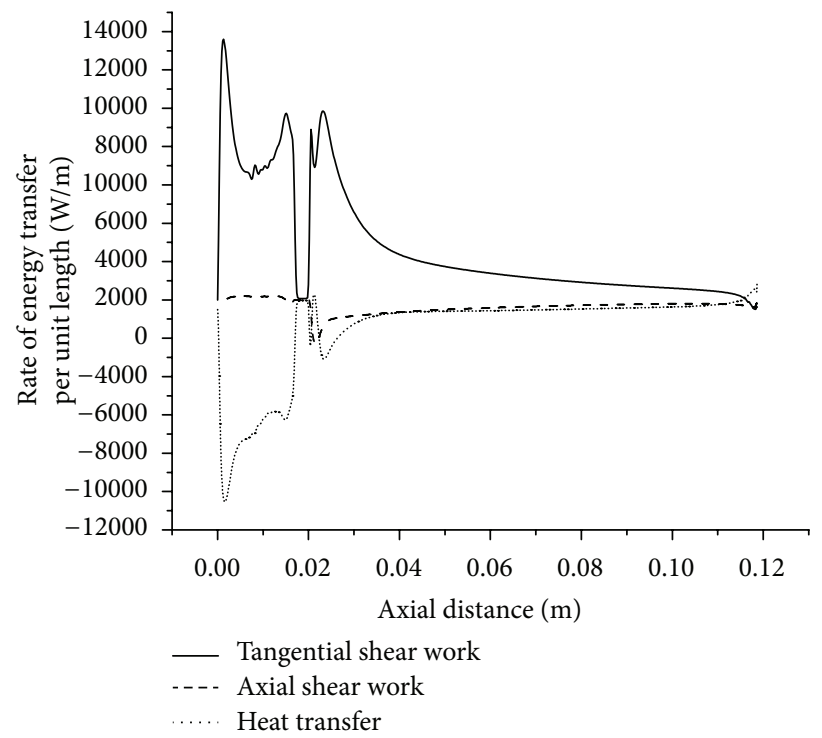

(b)

FIgURE 20: Rate of energy transfer per unit length $\left(\mathrm{Wm}^{-1}\right)$ across the control surfaces at $r=5 \mathrm{~mm}$ for (a) $\zeta=0 \mathrm{~mm}$, (b) $\zeta=18 \mathrm{~mm}$.
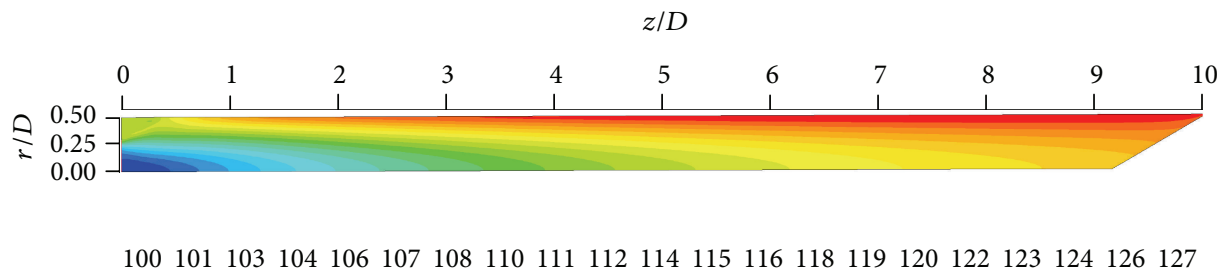

(a)

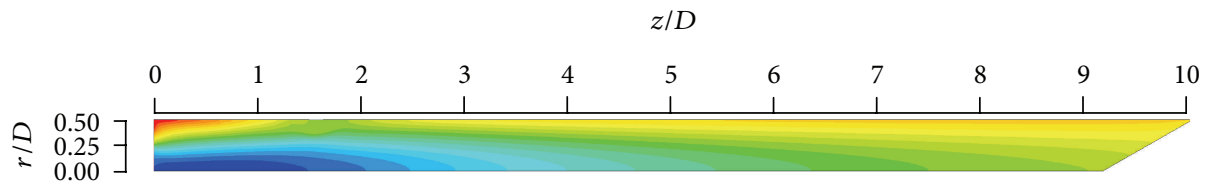

$\begin{array}{lllllllllllllllllllll}98 & 100 & 101 & 103 & 105 & 106 & 108 & 110 & 111 & 113 & 115 & 116 & 118 & 120 & 121 & 123 & 125 & 126 & 128 & 130 & 131\end{array}$

(b)

Figure 21: Total temperature (K) distribution for (a) $\zeta=0 \mathrm{~mm}$, (b) $\zeta=18 \mathrm{~mm}$.

significantly. However, the improvement in energy separation becomes small for pressures higher than $0.7 \mathrm{MPa}(\mathrm{abs})$.

(vi) Energy separation increases with the $L / D$ at cryogenic temperature. However, beyond $L / D=12.5$, improvement in energy separation becomes insignificant. It may be noted that the critical $L / D$ was found to be 30 for VTs operating with air at normal atmospheric temperature [10].

(vii) Inlet nozzles should be located closest to the cold outlet plane to achieve higher energy separation. (viii) CFD simulation with different gases as working fluids shows that the gas having higher value of the factor $c_{p} M$ produces lower energy separation. Of all gases investigated in this study, argon produces the maximum energy separation.

\section{Nomenclature}

$c_{p}$ : Specific heat at constant pressure $\left(\mathrm{Jkg}^{-1} \mathrm{~K}^{-1}\right)$

$D$ : Diameter of the VT $(\mathrm{m})$

$k$ : Thermal conductivity $\left(\mathrm{Wm}^{-1} \mathrm{k}^{-1}\right)$ 


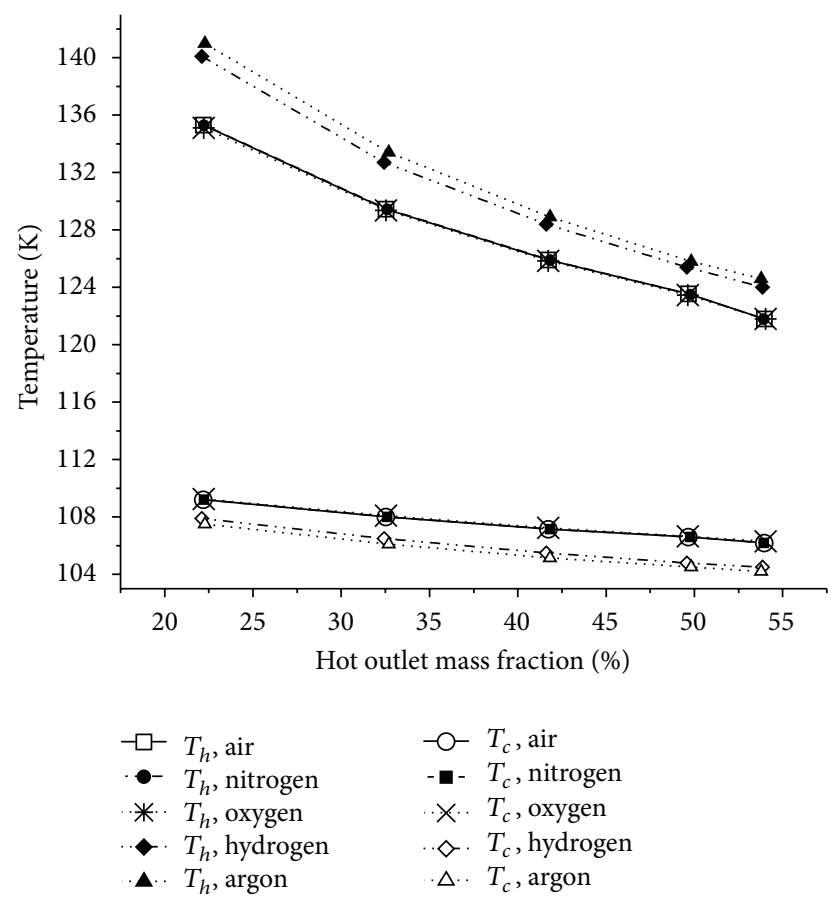

FIGURE 22: Hot and cold outlet temperatures (K) for different working fluids.

\section{$L$ : Length of the VT (m) \\ $M$ : Molecular weight $\left(\mathrm{kgkmol}^{-1}\right)$ \\ $p$ : Pressure $(\mathrm{Pa})$ \\ $r$ : Radial coordinate (m) \\ T: Temperature (K) \\ $u$ : Swirl velocity $\left(\mathrm{ms}^{-1}\right)$ \\ $v: \quad$ Radial velocity $\left(\mathrm{ms}^{-1}\right)$ \\ $w$ : Axial velocity $\left(\mathrm{ms}^{-1}\right)$ \\ $z$ : Axial coordinate $(\mathrm{m})$.}

\section{Greek Symbols}

$\varepsilon:$ Turbulent dissipation rate $\left(\mathrm{m}^{2} \mathrm{~s}^{-3}\right)$

$\theta$ : Angular coordinate (radian)

$\kappa:$ Turbulent kinetic energy $\left(\mathrm{m}^{2} \mathrm{~s}^{-2}\right)$

$\mu$ : Dynamic viscosity $\left(\mathrm{kgm}^{-1} \mathrm{~s}^{-1}\right)$.

\section{Subscripts}

c: Cold outlet

eff: Effective

$h$ : Warm outlet

$i$ : Inlet.

\section{References}

[1] G. J. Ranque, "Experiments on expansion in a vortex with simultaneous exhaust of hot air and cold air," Le Journal de Physique et le Radium, vol. 4, pp. 112-114, 1933.

[2] R. Hilsch, "The use of the expansion of gases in a centrifugal field as cooling process," Review of Scientific Instruments, vol. 18, no. 2, pp. 108-113, 1947.
[3] Y. Xue, M. Arjomandi, and R. Kelso, "A critical review of temperature separation in a vortex tube," Experimental Thermal and Fluid Science, vol. 34, no. 8, pp. 1367-1374, 2010.

[4] C. D. Fulton, "Ranque's tube," Journal of the ASRE: Refrigerating Engineering, vol. 58, pp. 473-479, 1950.

[5] C. U. Linderstrom-Lang, "The three-dimensional distributions of tangential velocity and total-temperature in vortex tubes," Journal of Fluid Mechanics, vol. 45, no. 1, pp. 161-187, 1971.

[6] M. Kurosaka, "Acoustic streaming in swirling flows," Journal of Fluid Mechanics, vol. 124, pp. 139-172, 1982.

[7] B. Ahlborn and S. Groves, "Secondary flow in a vortex tube," Fluid Dynamics Research, vol. 21, no. 2, pp. 73-86, 1997.

[8] M. H. Saidi and M. S. Valipour, "Experimental modeling of vortex tube refrigerator," Applied Thermal Engineering, vol. 23, no. 15, pp. 1971-1980, 2003.

[9] N. F. Aljuwayhel, G. F. Nellis, and S. A. Klein, "Parametric and internal study of the vortex tube using a CFD model," International Journal of Refrigeration, vol. 28, no. 3, pp. 442-450, 2005.

[10] U. Behera, P. J. Paul, S. Kasthurirengan et al., "CFD analysis and experimental investigations towards optimizing the parameters of Ranque-Hilsch vortex tube," International Journal of Heat and Mass Transfer, vol. 48, no. 10, pp. 1961-1973, 2005.

[11] O. V. Kazantseva, S. A. Piralishvili, and A. A. Fuzeeva, "Numerical simulation of swirling flows in vortex tubes," High Temperature, vol. 43, no. 4, pp. 608-613, 2005.

[12] S. Eiamsa-ard and P. Promvonge, "Numerical investigation of the thermal separation in a Ranque-Hilsch vortex tube," International Journal of Heat and Mass Transfer, vol. 50, no. 5-6, pp. 821-832, 2007.

[13] T. Farouk and B. Farouk, "Large eddy simulations of the flow field and temperature separation in the Ranque-Hilsch vortex tube," International Journal of Heat and Mass Transfer, vol. 50, no. 23-24, pp. 4724-4735, 2007.

[14] S. Eiamsa-Ard and P. Promvonge, "Numerical simulation of flow field and temperature separation in a vortex tube," International Communications in Heat and Mass Transfer, vol. 35, no. 8, pp. 937-947, 2008.

[15] T. Farouk, B. Farouk, and A. Gutsol, "Simulation of gas species and temperature separation in the counter-flow Ranque-Hilsch vortex tube using the large eddy simulation technique," International Journal of Heat and Mass Transfer, vol. 52, no. 13-14, pp. 3320-3333, 2009.

[16] A. Secchiaroli, R. Ricci, S. Montelpare, and V. D’Alessandro, "Numerical simulation of turbulent flow in a Ranque-Hilsch vortex tube," International Journal of Heat and Mass Transfer, vol. 52, no. 23-24, pp. 5496-5511, 2009.

[17] T. Dutta, K. P. Sinhamahapatra, and S. S. Bandyopdhyay, "Comparison of different turbulence models in predicting the temperature separation in a Ranque-Hilsch vortex tube," International Journal of Refrigeration, vol. 33, no. 4, pp. 783-792, 2010.

[18] T. Dutta, Experimental investigation and cfd analysis of energy separation in Ranque-Hilsch vortex tube [Ph.D. thesis], IIT Kharagpur, Kharagpur, India, 2011.

[19] R. Shamsoddini and A. H. Nezhad, "Numerical analysis of the effects of nozzles number on the flow and power of cooling of a vortex tube," International Journal of Refrigeration, vol. 33, no. 4, pp. 774-782, 2010.

[20] S. Eiamsa-ard, K. Wongcharee, and P. Promvonge, "Experimental investigation on energy separation in a counter-flow 
Ranque-Hilsch vortex tube: effect of cooling a hot tube," International Communications in Heat and Mass Transfer, vol. 37, no. 2, pp. 156-162, 2010.

[21] J. R. Simões-Moreira, "An air-standard cycle and a thermodynamic perspective on operational limits of Ranque-Hilsh or vortex tubes," International Journal of Refrigeration, vol. 33, no. 4, pp. 765-773, 2010.

[22] F. Kocabas, M. Korkmaz, U. Sorgucu, and S. Donmez, "Modeling of heating and cooling performance of counter flow type vortex tube by using artificial neural network," International Journal of Refrigeration, vol. 33, no. 5, pp. 963-972, 2010.

[23] T. Dutta, K. P. Sinhamahapatra, and S. S. Bandyopadhyay, "Numerical investigation of gas species and energy separation in the Ranque-Hilsch vortex tube using real gas model," International Journal of Refrigeration, vol. 34, no. 8, pp. 2118-2128, 2011.

[24] V. Balepin and D. Rosholt, "Progress in air separation with the vortex tube," in Proceedings of the 34th Joint Propulsion Conference and Exhibit, AIAA-98-3776, Cleveland, Ohio, USA, 1998.

[25] V. Balepin, D. Rosholt, and D. Petley, "Progress in air separation with the vortex tube," in Proceedings of the 9th International Space Planes and Hypersonic systems and Technologies Conference, AIAA-99-4844, Norfolk, Va, USA, 1999.

[26] A. M. Crocker, S. M. White, and F. Bremer Jr., "Experimental results of a vortex tube air separator for advanced space transportation," in Proceedings of the 39th Joint Propulsion Conference and Exhibit, AIAA-2003-4451, Huntsville, Ala, USA, 2003.

[27] A. M. Crocker, G. L. Sutphin, D. V. Cassisi, C. Knowlen, and R. F. Weimer, "Investigation of enhanced vortex tube air separators for advanced space transportation," in Proceedings of the 40th AIAA/ASME/SAE/ASEE Joint Propulsion Conference and Exhibit, AIAA 2004-3392, Fort Lauderdale, Fla, USA, July 2004.

[28] J. Y. Liu, M. Q. Gong, J. F. Wu, Y. Cao, and E. C. Luo, "An experimental research on a small flow vortex tube at low temperature ranges," in Proceedings of the 20th International Cryogenic Engineering Conference (ICEC '04), pp. 165-168, Beijing, China, May 2004.

[29] E. W. Lemmon, M. O. McLinden, and M. L. Huber, REFPROP_Reference Fluid Thermodynamic and Transport Properties, NIST Standard Reference Database 23, Version 7.0, National Institute of Standards and Technology, Gaithersburg, Md, USA, 2002.

[30] U. Behera, P. J. Paul, K. Dinesh, and S. Jacob, "Numerical investigations on flow behaviour and energy separation in Ranque-Hilsch vortex tube," International Journal of Heat and Mass Transfer, vol. 51, no. 25-26, pp. 6077-6089, 2008.

[31] R. Kassner and E. Knoernschild, "Friction laws and energy transfer in circular flow, Tech. Rep. F-TR-2198ND, WrightPatterson Air Force Base, Dayton, Ohio, USA, 1948.

[32] A. J. Reynolds, "Energy flows in a vortex tube," Zeitschrift für Angewandte Mathematik und Physik, vol. 12, no. 4, pp. 343-357, 1961.

[33] W. Fröhlingsdorf and H. Unger, "Numerical investigations of the compressible flow and the energy separation in the RanqueHilsch vortex tube," International Journal of Heat and Mass Transfer, vol. 42, no. 3, pp. 415-422, 1998.

[34] S. A. Colgate and J. R. Buchler, "Coherent transport of angular momentum. The Ranque-Hilsch tube as a paradigm," Annals of the New York Academy of Sciences, vol. 898, pp. 105-112, 2000. 

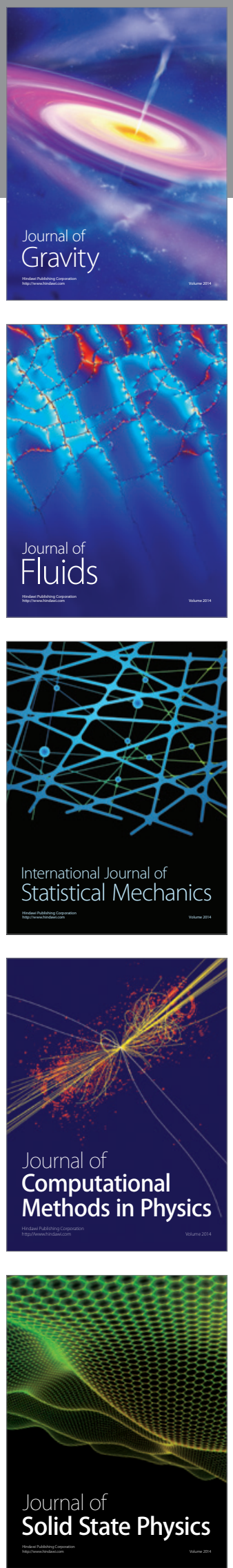

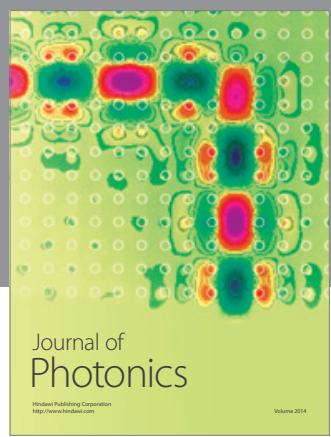

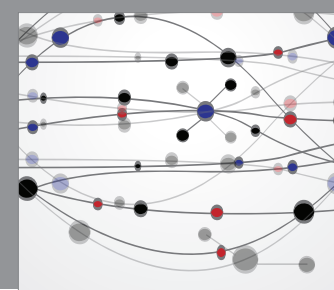

The Scientific World Journal

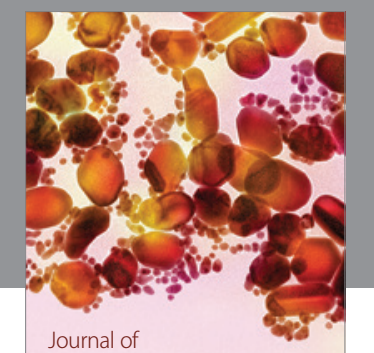

Soft Matter
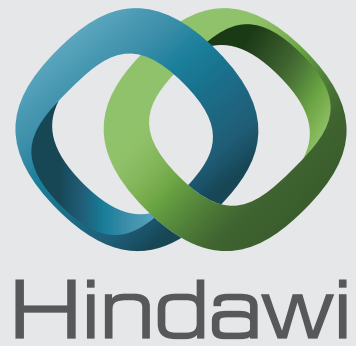

Submit your manuscripts at

http://www.hindawi.com
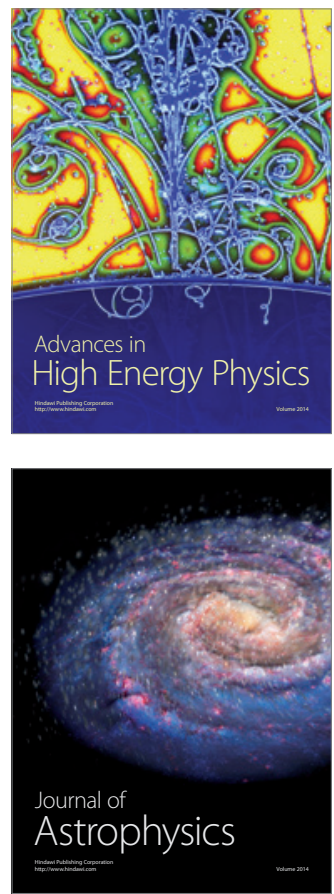
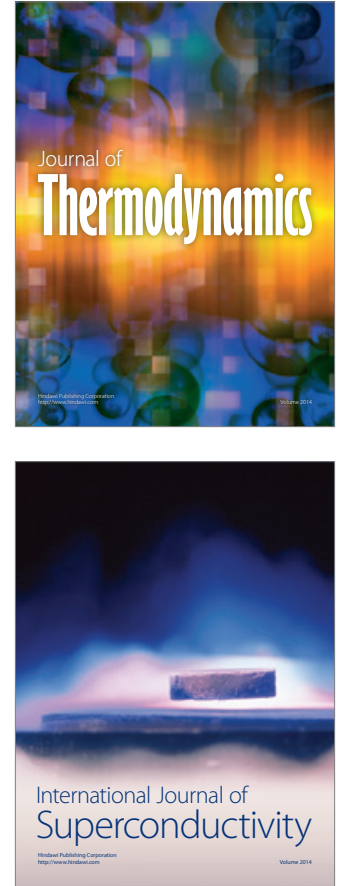
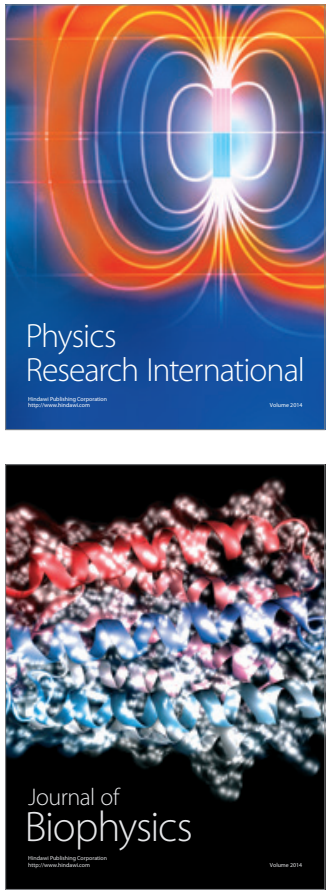
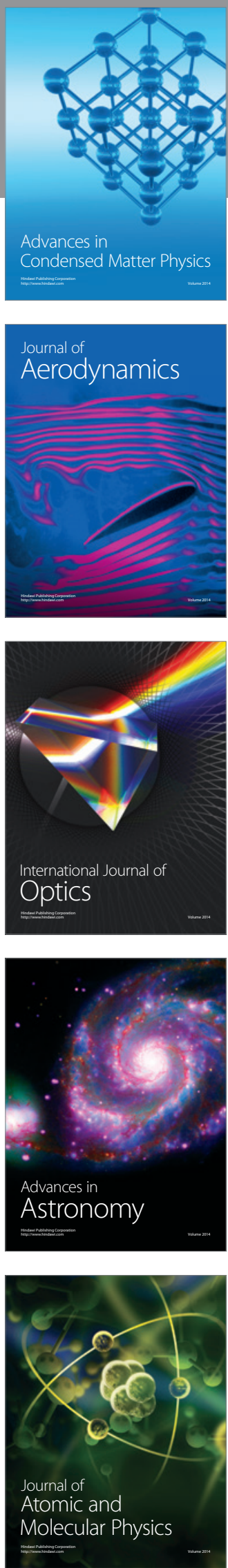